\title{
Compostos à base de molibdênio para remediação ambiental: uma revisão
}

\author{
Molybdenum-based compounds for environmental remediation: a review \\ Compuestos a base de molibdeno para la remediación ambiental: una revisión
}

\author{
Carlos Alves do Nascimento Filho \\ ORCID: https://orcid.org/0000-0002-9237-3271 \\ Universidade Federal do Vale do São Francisco, Brasil \\ E-mail: carlosfilho85@hotmail.com \\ Helinando Pequeno de Oliveira \\ ORCID: https://orcid.org/0000-0002-7565-5576 \\ Universidade Federal do Vale do São Francisco, Brasil \\ E-mail: helinando.oliveira@univasf.edu.br
}

\begin{abstract}
Resumo
O dissulfeto de molibdênio é um material amplamente utilizado para remediação ambiental tendo em vista sua excelente capacidade de adsorção, que é atribuída principalmente aos sítios ativos de enxofre na superfície do $\mathrm{MoS}_{2}$. Além disso, este material possui vantagens quando comparado com outros fotocatalisadores, como por exemplo por alta atividade fotocatalítica, baixa toxicidade e boa capacidade de remoção de contaminantes orgânicos e inorgânicos. Nesta revisão apresentaremos os diferentes métodos de preparação do dissulfeto de molibdênio a partir da esfoliação mecânica, química, eletroquímica e os métodos hidrotérmico, solvotérmico e de deposição de vapor químico. Abordaremos ainda suas propriedades tais como as capacidades de adsorção para diferentes tipos de metais pesados em solução, os tipos de degradação fotocatalítica a partir da comparação dos adsorventes a base de $\mathrm{MoS}_{2}$ com outros adsorventes, os mecanismos de adsorção e os fatores que afetam esse processo, como o pH e temperatura da solução, tempo de contato, tipos de contaminantes e a influência de outros íons presentes em solução que podem atrapalhar o processo de adsorção. Outro processo a ser reportado se refere à associação entre o $\mathrm{MoS}_{2}$ e outros compostos, como óxido de grafeno e compostos derivados do nitrogênio, óxido de titânio e associações com bismuto e prata, tendo como principal vantagem o aumento da capacidade de adsorção do material. Como conclusão, será tratado sobre a eficiência de remoção das diferentes associações do dissulfeto de molibdênio frente aos diferentes tipos de contaminantes assim como os diversos fatores que influenciam nesta eficiência.
\end{abstract}

Palavras-chave: Dissulfeto de molibdênio; Remediação ambiental; Adsorção.

\begin{abstract}
Molybdenum disulfide is a widely used material for environmental remediation in view of its excellent adsorption capacity, which is attributed to the active sulfur sites on the $\mathrm{MoS}_{2}$ surface. In addition, it presents advantages in comparison with other photocatalysts, such as high photocatalytic activity, low toxicity and good ability to remove organic and inorganic contaminants. In this review we will present the different methods of preparing molybdenum disulfide from mechanical, chemical, electrochemical exfoliation and the hydrothermal, solvothermal and chemical vapor deposition methods. It will be also addressed about its superior properties such as the adsorption capacities for different types of heavy metals in solution, the types of photocatalytic degradation from the comparison with $\mathrm{MoS}_{2}$ based adsorbents with other adsorbents, as well as the adsorption mechanisms and the factors that affect this process, such as the $\mathrm{pH}$ and temperature of the solution, contact time, types of contaminants as well as the influence of other ions present in the solution that can hinder the adsorption process. The association of $\mathrm{MoS}_{2}$ with compounds based on graphene oxide and derived from nitrogen, titanium oxide and associations with bismuth and silver introduces advantage of increasing the material's ability to be explored as an adsorbent. Moreover, it is reported about the removal efficiency of the different associations of molybdenum disulfide against the different types of contaminants as well as the different factors that affect the overall efficiency.
\end{abstract}

Keywords: Molybdenum disulfide; Environmental remediation; Adsorption.

\section{Resumen}

El disulfuro de molibdeno es un material ampliamente utilizado para la remediación ambiental en vista de su excelente capacidad de adsorción, que se atribuye principalmente a los sitios activos de azufre en la superficie de $\mathrm{MoS}_{2}$. Además, tiene ventajas en comparación con otros fotocatalizadores, como por ejemplo debido a su alta actividad fotocatalítica, baja toxicidad y buena capacidad para eliminar contaminantes orgánicos e inorgánicos. En esta revisión presentaremos los diferentes métodos de preparación de disulfuro de molibdeno a partir de exfoliación mecánica, química, electroquímica y los métodos de deposición de vapor hidrotermal, solvotermal y químico. 
También abordaremos sus propiedades como las capacidades de adsorción para diferentes tipos de metales pesados en solución, los tipos de degradación fotocatalítica a partir de la comparación de adsorbentes basados en $\mathrm{MoS}_{2}$ con otros adsorbentes, los mecanismos de adsorción y los factores que afectan este proceso, tales como el pH y la temperatura de la solución, el tiempo de contacto, los tipos de contaminantes y la influencia de otros iones presentes en la solución que pueden dificultar el proceso de adsorción. Otro proceso a reportar se refiere a la asociación entre $\mathrm{MoS}_{2}$ y otros compuestos, como el óxido de grafeno y compuestos derivados de nitrógeno, óxido de titanio y asociaciones con bismuto y plata, con la principal ventaja de incrementar la capacidad de adoración del material. Como conclusión, hablaremos de la eficiencia de remoción de las diferentes asociaciones de disulfuro de molibdeno frente a los diferentes tipos de contaminantes, así como de los diferentes factores que influyen en esta eficiencia.

Palabras clave: Disulfuro de molibdeno; Remediación ambiental; Adsorción.

\section{Introdução}

O crescimento da população global e a industrialização têm graves consequências sobre a degradação ambiental. A contaminação do solo e da água por metais pesados tem levado a uma grande crise mundial, visto que vários contaminantes são nocivos à saúde dos seres vivos (Shahzad et al., 2020). A contaminação por íons como chumbo, cádmio, mercúrio, cobre, cromo e níquel causa graves problemas nas cadeias alimentares por causa da bioacumulação. Mesmo assim, quantidades crescentes de metais pesados vem sendo descartada em rios e mares (Luo et al., 2019). Outro contaminante amplamente discutido e caracterizado na literatura é o mercúrio, um dos metais pesados mais tóxicos para organismos vivos que está presente na água na forma de íons e em vias aéreas na forma neutra $\left(\mathrm{Hg}^{0}\right)$ (Shahzad et al., 2020) decorrente de processos de mineração.

A concentração máxima desse elemento que é permitida pela Organização Mundial da Saúde (OMS) na água potável é de $1 \mu \mathrm{g} / \mathrm{L}$ e em águas residuais esse valor sobe para $5 \mu \mathrm{g} / \mathrm{L}$. A poluição por este elemento é originária de indústrias, minas, processamento de materiais metálicos não ferrosos, mas é a combustão do carvão mineral em escala industrial o maior contribuinte (34\%) de mercúrio em escala global (Shahzad et al., 2020).

Outro detalhe importante se refere à presença de diferentes metais pesados e com diferentes íons (como por exemplo $\mathrm{Ni}^{2+}$ e $\mathrm{CrO}_{4}{ }^{2-}$ ) em amostras de água e de efluentes. A completa remoção destes contaminantes se dá por suas propriedades químicas intrínsecas. Portanto, é urgente é de fundamental importância explorar os mecanismos de remoção simultânea de compostos catiônicos e aniônicos para identificar o desempenho competitivo ou sinérgico entre os dois tipos de íons e também entre os diferentes tipos de adsorventes (Cai et al., 2020). Esta condição requer tecnologias eficientes para remover os diferentes tipos de contaminantes que podem estar presentes na água e/ou efluentes. Em comparação com outras tecnologias, a adsorção é considerada uma tecnologia promissora para este propósito (Lee et al., 2016). Vários adsorventes, incluindo argilas, carvão ativado, biomassa e nanotubos de carbono têm sido aplicados para remover o cromo $\left(\mathrm{Cr}^{3+} \mathrm{ou} \mathrm{Ni}^{2+}\right)$, contudo, esses adsorventes frequentemente exibem baixa seletividade e pouca afinidade de ligação em relação a um determinado contaminante específico ao tratar águas residuais contendo componentes complexos (Cai et al., 2020).

Nesse contexto, os adsorventes devem ser capazes de interagir com os diferentes poluentes e apresentar boa especificidade para fins de bom desempenho na remoção destes poluentes específicos. Por causa da grande afinidade entre íons de metais pesados e enxofre através de interações eletrostáticas, compósitos contendo grupos sulfonados têm sido explorados e demonstraram ser excelentes materiais para remover íons de metais pesados (Cai et al., 2020). Materiais contendo sílica funcionalizada com grupos tiol, nanofibras de celulose modificadas com grupos tiol, carvão ativado e óxido de grafeno

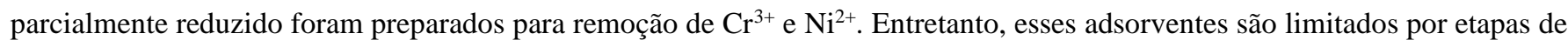
preparação complexas. Além disso, os mesmos apresentam capacidades de adsorção baixas, devido principalmente ao baixo teor de enxofre em sua composição (Cai et al., 2020). Diante disso, se faz necessário desenvolver novos adsorventes que sejam mais fáceis de sintetizar e que tenham maiores capacidades de adsorção, tais como o dissulfeto de molibdênio, um material 
promissor que é amplamente utilizado para a remediação ambiental. $\mathrm{O} \mathrm{MoS}_{2}$ é um sólido preto prateado que ocorre naturalmente na forma do mineral molibdenita, sendo o principal minério para o molibdênio. É utilizado em baterias de íon de lítio, supercapacitores, fototransistores, liberação de fármacos, geração de hidrogênio, etc. (Yuan et al., 2016). Além disso, o $\mathrm{MoS}_{2}$ possui sítios ativos que podem adsorver vários poluentes e, portanto, serem utilizados para remediação ambiental (Kumar et al., 2019).

Além destas aplicações, o $\mathrm{MoS}_{2}$ vem sendo usado na degradação fotocatalítica de contaminantes orgânicos, inativação de bactérias (Fausey et al., 2020) e na remoção de metais pesados como prata, cádmio, chumbo, mercúrio, cromo, níquel, contaminantes orgânicos em água e efluentes (Luo et al., 2019). $\mathrm{O} \mathrm{MoS}_{2}$ pode ser obtido por meio de vários métodos sintéticos, tendo conformações variadas como nanofolhas, formato de flor, camadas lamelares, sempre levando a diferentes desempenhos de adsorção para diferentes materiais analisados (Luo et al., 2019). Com isso, a alta razão superfície-volume e a boa capacidade de adsorção/absorção tornam este um material promissor a ser aplicado na a remoção de mercúrio, com cinética rápida e excelente seletividade (Shahzad et al., 2020), sendo capaz de adsorver $\mathrm{Cr}^{6+} \mathrm{e} \mathrm{Ni}^{2+}$ devido ao seu alto teor de enxofre, que possui interações com diversos contaminantes metálicos. Cada monocamada de $\mathrm{MoS}_{2}$ é composta por três camadas de átomos covalentemente ligados como enxofre-molibdênio-enxofre. A espessura das três camadas S-Mo-S de $\mathrm{MoS}_{2}$ é de cerca de 0,3 nm e essa largura ajuda a reter íons de diversos tipos em sua rede (Cai et al., 2020). Diante disso, a disponibilidade de átomos de enxofre é crucial para que o $\mathrm{MoS}_{2}$ possa remover com eficiência os poluentes visto que os átomos de enxofre são os principais locais de adsorção desse composto (Cai et al., 2020). Estudos têm mostrado que as camadas lamelares do dissulfeto de molibdênio são ativas e que o enxofre pode agir como base para formar o complexos com íons $\mathrm{Hg} / \mathrm{Pb} / \mathrm{Cd} / \mathrm{Cr} / \mathrm{Ni}$ entre outros através interações eletrostáticas e ligações covalentes (Su et al., 2019). Neste sentido, o objetivo deste estudo é reunir, organizar e fornecer informações contidas na literatura recente e que trata do estado-da-arte sobre as diferentes formas de síntese do dissulfeto de molibdênio assim como as suas diferentes associações, identificando os pontos principais (positivos e negativos de cada síntese e associação), como forma de identificar aspectos importantes pouco explorados cientificamente e as novas fronteiras a serem abordadas para o tema.

\section{Metodologia}

Este estudo constitui uma revisão bibliográfica documental de caráter analítico e qualitativo do tipo revisão integrativa, que busca reunir o conhecimento recente e o estado-da -arte acerca do uso do dissulfeto de molibdênio em remediação ambiental. Esta constatação se deu pelo número crescente de publicações na área, o que viabilizou este olhar integrativo sobre diferentes metodologias que completam o conhecimento sobre a técnica. A coleta de dados foi realizada no período de agosto/2020 a dezembro/2020 e utilizou-se para a pesquisa as bases de dados Web of Science - Coleção Principal (Clarivate Analytics), Scientific Eletronic Library Online (SCIELO) e National Library of Medicine (PUBMED).

\section{Resultados e Discussão}

O dissulfeto de molibdênio possui camadas lamelares interligadas entre os átomos de molibdênio e os átomos de enxofre, sendo que esses átomos são conectados por ligações covalentes (Chang \& Chen, 2011) com as interações entre os planos entre os planos lamelares por forças de van der Waals (Liu et al., 2019). O comprimento da ligação entre molibdênio e enxofre é de 2,4 ̊̊ e a distância entre os átomos de enxofre (superiores e inferiores) é igual a 3,1 Å (Wu et al., 2018). A Figura 1 mostra as camadas lamelares do dissulfeto de molibdênio, na qual os interstícios, defeitos e vacâncias são utilizados para dopar o material, estando os átomos de enxofre localizados nas bordas da estrutura, o que é relevante para a estrutura, dado que os mesmos são significativamente ativos. 
Figura 1: Camadas lamelares do dissulfeto de molibdênio.

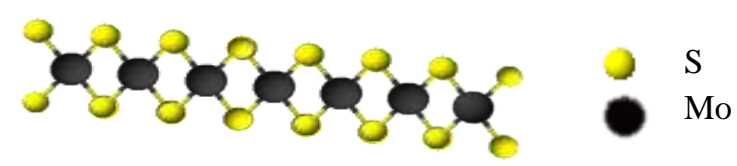

Fonte: Autores (2021).

Dependendo do empilhamento de átomos em cada monocamada, é possível formar diferentes estruturas de cristal de $\mathrm{MoS}_{2}$ : ao considerar a fase $2 \mathrm{H}$ semicondutora termodinamicamente estável ou a metaestável, com coordenação trigonal e 2 camadas lamelares. A fase 3R também exibe coordenação trigonal mas com 3 camadas lamelares. Já a fase 1 T é octaédrica e metálica (Wu et al., 2018). A Figura 2 mostra as fases cristalina 2 H, 3 R e 1 T onde é perceptível que a diferença entre elas é o parâmetro de rede $c$.

Figura 2: Fases cristalinas 2H, 3R e $1 \mathrm{~T}$ do $\mathrm{MoS}_{2}$.
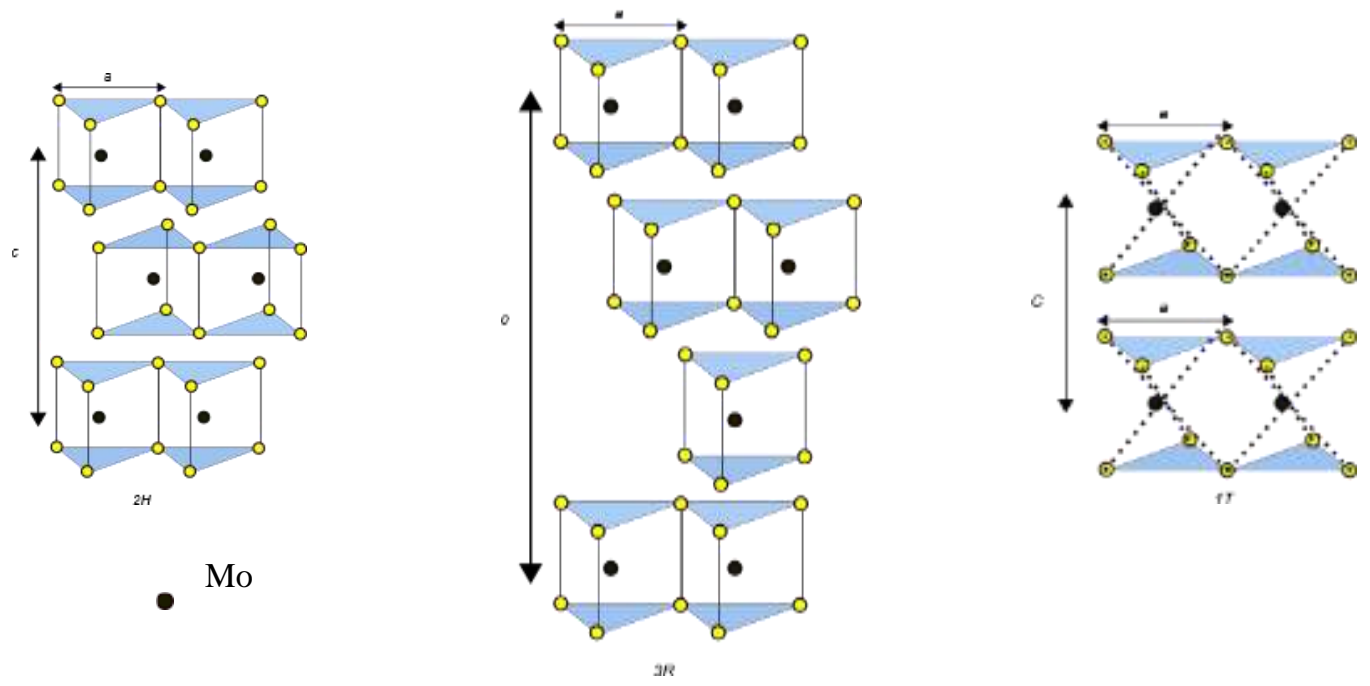

Fonte: Autores (2021).

As três fases podem se transformar de uma para outra em condições especiais. As fases $1 \mathrm{~T}-\mathrm{MoS}_{2}$ e $3 \mathrm{R}-\mathrm{MoS}_{2}$ se transformarão na fase $2 \mathrm{H}-\mathrm{MoS}_{2}$ após recozimento e a fase $2 \mathrm{H}-\mathrm{MoS}_{2}$ pode se transformar em $1 \mathrm{~T}-\mathrm{MoS}_{2}$ pela inserção de íons de lítio ou bombardeio por elétrons durante a esfoliação (Wu et al., 2018). O controle na estrutura cristalina e em propriedades físico-químicas distintas do $\mathrm{MoS}_{2}$ pode ser obtido por meio de vários métodos sintéticos, o que leva a desempenhos de adsorção diferentes em remoção de metais pesados, de contaminantes e inativação de bactérias (Luo et al., 2019), como descrito a seguir.

\subsection{Preparação do $\mathrm{MoS}_{2}$}

\subsubsection{Esfoliação mecânica}

A esfoliação mecânica é um método simples para obtenção de monocamadas de diferentes materiais, útil na remoção mecânica de camadas do material até o ponto em que é obtida uma monocamada. No entanto, este método não é aplicável para a preparação de $\mathrm{MoS}_{2}$ como adsorvente devido ao seu baixo rendimento e defeitos mínimos na superfície. 
A esfoliação mecânica por fita adesiva pode gerar nanofolhas de $\mathrm{MoS}_{2}$ grandes, limitadas por defeitos e de grau eletrônico para estudos fundamentais e demonstrações de dispositivos eletrônicos (Wang \& Mi, 2017).

\subsubsection{Esfoliação líquida}

A técnica da esfoliação líquida envolve a esfoliação mecânica de cristais lamelares por ultrassonicação (ou cisalhamento) em meio líquido (Hernandez et al., 2008).

A partir da técnica de esfoliação baseada nos grafenos o pó de $\mathrm{MoS}_{2}$ é misturado a compostos tensoativos para garantir que a tensão superficial do líquido seja correspondente à energia livre superficial do dissulfeto de molibdênio. Alguns solventes como como N-metil-pirrolidona (NMP) e dimetilsulfóxido (DMSO) são comumente usados para a esfoliação líquida de $\mathrm{MoS}_{2}$ (Coleman et al., 2011).

O ultrassom é a técnica geralmente utilizada para este processo, visto que as ligações entre os planos são fracas (do tipo van de Waals), diferentemente das ligações existentes entre os átomos (ligação covalente forte). A força aplicada também atua sobre as camadas para reduzir o tamanho da folha, que são menores que as nanofolhas produzidas pela esfoliação mecânica (Jawaid et al., 2016).

De forma geral, nanofolhas de $\mathrm{MoS}_{2}$ com uma menor quantidade de camadas podem ser separadas na suspensão a partir de processos de centrifugação. O método de esfoliação líquida é um processo que pode fabricar a fase $2 \mathrm{D}-\mathrm{MoS}_{2} \mathrm{em}$ uma escala maior em comparação com a esfoliação mecânica (Jawaid et al., 2016).

Entretanto, essa técnica possui baixo rendimento, pois apenas o sobrenadante é armazenado para evitar a maioria dos sedimentos que inevitavelmente resultam em um baixo rendimento (Tan et al., 2019).

\subsubsection{Esfoliação eletroquímica}

$\mathrm{MoS}_{2}$ também pode ser preparado por meio de esfoliação eletroquímica. Neste processo, o $\mathrm{MoS}_{2}$ é usado como catodo ou anodo enquanto que o outro eletrodo é uma folha de platina sendo ambos mergulhados em uma solução salina. Uma tensão elétrica é aplicada no sistema promovendo o crescimento do $\mathrm{MoS}_{2}$.

Nesse processo, a água se decompõe em hidrogênio e oxigênio, que fluem da solução para o ar através dos eletrodos, o que leva à expansão do volume de $\mathrm{MoS}_{2}$, produzindo monocamadas semicondutoras desse material (Liu et al., 2017).

Para ter um aumento no rendimento de formação de monocamadas de $\mathrm{MoS}_{2}$, Zeng e seu grupo de pesquisa desenvolveram um método eletroquímico através da intercalação de lítio. Neste método, ao invés de se usar uma folha de platina no anodo é usada uma folha de lítio, tendo uma configuração semelhante a uma bateria de íons lítio (Zeng et al., 2011).

Durante o processo de carga, os íons lítio entram nos planos lamelares do $\mathrm{MoS}_{2}$, ampliando dessa forma o espaçamento interlamelar. Durante o processo de descarga e através de sonicação, o lítio intercalado nas camadas lamelares reage com água, ocorrendo a formação de hidrogênio, o que ajuda a formar uma suspensão dispersa de nanofolhas de $\mathrm{MoS}_{2}$ (Zeng et al., 2011).

\subsubsection{Método hidrotérmico}

Nesse método, o dissulfeto de molibdênio é sintetizado através da reação entre os precursores iniciais de molibdênio e enxofre. Nesta síntese são geralmente utilizados o molibdato de amônio ou molibdato de sódio como precursores de molibdênio e um composto tiosulfonado como precursor de enxofre (Ma et al., 2009). Para síntese do $\mathrm{MoS}_{2}$ com aspecto parecido ao de uma flor, os precursores de Mo e S são primeiramente dispersos em água, que é aquecida em autoclave selada a $220^{\circ} \mathrm{C}$ por $6 \mathrm{~h}$. 
O dissulfeto de molibdênio preparado pelo método hidrotérmico possui mais defeitos estruturais em sua rede com uma maior a quantidade de enxofre na estrutura, o que torna a quantidade de contaminantes adsorvidos, visto que há mais sítios ativos na estrutura e esses defeitos podem ser aumentados pela introdução de mais enxofre nos precursores iniciais (Xie et al., 2014).

Outro detalhe é que é relativamente fácil introduzir metais, grupos funcionais e outros materiais nos interstícios, defeitos e vacâncias da estrutura (Song et al., 2015). Entretanto, a síntese hidrotérmica não permite o controle preciso da quantidade de camadas lamelares do dissulfeto de molibdênio, podendo ocorrer superposição de camadas, o que afeta a morfologia final do material sintetizado (Shi et al., 2012).

\subsubsection{Método solvotérmico}

Esse método foi desenvolvido tendo por base o método hidrotérmico e a diferença básica entre ambos é que no método solvotérmico os solventes utilizados são orgânicos. Sendo assim, os precursores iniciais de molibdênio e enxofre são adicionados em solventes orgânicos como N,N-dimetilformamida (DMF), 1-metil-2-pirrolidinona (NMP) e polietilenoglicol, formando soluções homogêneas que em seguida são autoclavadas geralmente por $24 \mathrm{~h}$. Após esse processo, a solução resultante é centrifugada e o material resultante é seco em estufa para obtenção do pó de $\mathrm{MoS}_{2}$ (Najmaei et al., 2013).

Este método possui como vantagens a menor aglomeração das partículas (o que facilita a esfoliação posterior), forma controlável de partícula e o dissulfeto de molibdênio obtido com esses solventes e com temperaturas menores do que as utilizadas no método hidrotérmico possuem maior área superficial (Peng et al., 2002).

\subsubsection{Método de deposição de vapor químico}

Esse método é um dos mais comuns para a síntese de $\mathrm{MoS}_{2}$ e é muito utilizado para obtenção de material com alta qualidade (Lee et al., 2012), sendo considerado um método ascendente para o crescimento de nanofolhas de dissulfeto de molibdênio. Para tanto, os reagentes iniciais precursores de molibdênio e enxofre são decompostos para formar respectivamente Mo e S, e em seguida, camadas de $\mathrm{MoS}_{2}$ são formadas em um suporte sob certas condições controladas (Lin et al., 2012), geralmente usando a sulfurização do $\mathrm{MoO}_{3}$ (Lee et al., 2012).

Neste processo, $\mathrm{MoO}_{3}$ e S são evaporados e posteriormente o vapor de enxofre entra em contato com o $\mathrm{MoO}_{3}$, que pode estar em um suporte de $\mathrm{SiO}_{2} / \mathrm{Si}$ (Lee et al., 2012), safira ou poliimida (Ahn et al., 2015) por meio de um fluxo de gás inerte, podendo ser o nitrogênio ou argônio. Com isso, ocorre a formação $\mathrm{MoS}_{2}$ que cresce nesse suporte (Lee et al., 2012).

A espessura e a morfologia do filme de dissulfeto de molibdênio sintetizados são controlados pelas condições reacionais e os filmes produzidos por esta técnica são altamente cristalinos. Entretanto, é produzida uma quantidade muito pequena de $\mathrm{MoS}_{2}$, sendo pouco viável a sua utilização para produção em larga escala (Lee et al., 2012). Na Tabela 1 são mostrados os métodos de síntese, a morfologia assim como as vantagens e desvantagens de cada método de síntese do dissulfeto de molibdênio. 
Tabela 1: Vantagens e desvantagens dos diferentes métodos de síntese do $\mathrm{MoS}_{2}$.

\begin{tabular}{|c|c|c|c|c|}
\hline $\begin{array}{l}\text { MÉTODO } \\
\text { DE } \\
\text { SÍNTESE }\end{array}$ & MORFOLOGIA & VANTAGENS & DESVANTAGENS & REF. \\
\hline $\begin{array}{l}\text { Esfoliação } \\
\text { mecânica }\end{array}$ & Nanofolhas & $\begin{array}{c}\text { Fácil de preparar; alta } \\
\text { qualidade das } \\
\text { nanopartículas }\end{array}$ & Baixo rendimento & $\begin{array}{l}\text { (Mak et } \\
\text { al., 2010) }\end{array}$ \\
\hline $\begin{array}{c}\text { Esfoliação } \\
\text { líquida }\end{array}$ & $\begin{array}{c}\text { Camadas } \\
\text { bidimensionais }\end{array}$ & $\begin{array}{l}\text { Fácil de preparar; alto } \\
\text { rendimento }\end{array}$ & $\begin{array}{c}\text { Camadas de estágios } \\
\text { múltiplos }\end{array}$ & $\begin{array}{l}\text { (Smith et } \\
\text { al., 2011) }\end{array}$ \\
\hline $\begin{array}{c}\text { Esfoliação } \\
\text { eletroquímica }\end{array}$ & Fibras & $\begin{array}{l}\text { Mais produtos de } \\
\text { camadas simples }\end{array}$ & $\begin{array}{c}\text { Camadas de estágios } \\
\text { múltiplos }\end{array}$ & $\begin{array}{c}\text { (Eda et al., } \\
\text { 2011) }\end{array}$ \\
\hline $\begin{array}{c}\text { Método } \\
\text { hidrotérmico }\end{array}$ & Nanofolhas & Fácil de preparar & $\begin{array}{l}\text { Alto consumo de } \\
\text { energia }\end{array}$ & $\begin{array}{l}\text { (Midya et } \\
\text { al., 2016) }\end{array}$ \\
\hline $\begin{array}{c}\text { Método } \\
\text { solvotérico }\end{array}$ & Forma de flor & Fácil de preparar & Baixo rendimento & $\begin{array}{c}\text { (Xie et al., } \\
\text { 2014) }\end{array}$ \\
\hline $\begin{array}{c}\text { Método de } \\
\text { deposição de } \\
\text { vapor químico }\end{array}$ & Nanopartículas & $\begin{array}{l}\text { Amostra cristalina e } \\
\text { de alta qualidade; } \\
\text { tamanho e espessura } \\
\text { controláveis }\end{array}$ & $\begin{array}{c}\text { Alto consumo de } \\
\text { energia }\end{array}$ & $\begin{array}{c}\text { (Lee et al., } \\
\text { 2012) }\end{array}$ \\
\hline
\end{tabular}

Fonte: Autores (2021).

\subsection{Propriedades}

O dissulfeto de molibdênio possui uma estrutura do tipo "sanduiche", com átomos de molibdênio e enxofre intercalados entre si. A quantidade de enxofre na estrutura oferece vários sítios de adsorção na estrutura. Com base em sua estrutura eletrônica (como ilustrado na Figura 3), o enxofre possui uma grande afinidade por metais pesados que agem como ácidos de Lewis (Wang \& Mi, 2017).

Figura 3: Incorporação de metais pesados a estrutura do $\mathrm{MoS}_{2}$.

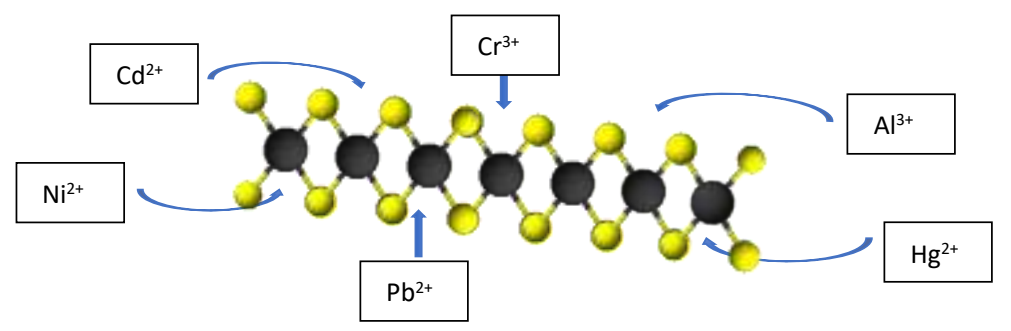

Fonte: Autores (2021).

Além destas vantagens, o composto apresenta potencial zeta negativo para todos os valores de pH, o que é benéfico para a interação/adsorção de diversos íons metálicos positivos (Jia et al., 2017). A superfície da base que é livre de defeitos é teoricamente neutra e as bordas que possuem maior concentração de molibdênio é negativa, devido a oxidação do mesmo (Jia et al., 2018), dessa forma, a incorporação de cátions na base do $\mathrm{MoS}_{2}$ diminui a carga negativa nas bordas do mesmo (região 
onde há maior densidade de carga negativa) devido à oxidação do molibdênio (Castro et al., 2016). As bordas possuem caráter hidrofílico enquanto que o plano basal possui propriedades hidrofóbicas. Essa hidrofobicidade juntamente com a neutralidade elétrica da superfície da base são desvantagens para o dissulfeto de molibdênio quando o mesmo é utilizado como adsorvente, devido a pequena taxa de dispersão e a fraca atração eletrostática. Estas desvantagens podem ser atenuadas pela introdução de defeitos, como interstícios, vacâncias e falhas no plano basal do $\mathrm{MoS}_{2}$ que mudam o balanço eletrostático da estrutura e facilitam o processo de adsorção/retenção de metais pesados e outros poluentes (Castro et al., 2016).

\subsection{Mecanismos de degradação fotocatalítica}

A degradação fotocatalítica de um poluente pode acontecer a partir da reação direta de oxidação entre este composto e uma vacância fotogerada ou da reação com os radicais oxidantes (HO•) formados pela reação entre estas vacâncias e as moléculas de água ou íons hidroxila $\left(\mathrm{OH}^{-}\right)$na superfície do semicondutor. A primeira rota reacional é denominada fotocatálise direta enquanto que a segunda é chamada de catálise indireta (Chen et al., 2014). Esse tipo de degradação aproveita radicais livres altamente ativos para "quebrar" o contaminante em pequenas moléculas através de reações de adição, substituição e transferência de elétrons entre radicais livres e poluentes orgânicos (Huang et al., 2017).

Para entender esse processo é necessário compreender como ocorre a condução dos fotoelétrons na interface do material, pois os contaminantes orgânicos reagem principalmente com as espécies reativas da superfície do mesmo (Li et al., 2015).

\subsubsection{Capacidade de adsorção de nanofolhas $\mathrm{MoS}_{2}$ para diferentes metais pesados}

Levando em consideração a estrutura bidimensional e a grande quantidade de átomos de enxofre expostos na estrutura, o $\mathrm{MoS}_{2}$ possui vários sítios de adsorção que são utilizados na captura de vários tipos de metais pesados e de outros poluentes.

$\mathrm{O} \mathrm{MoS}_{2}$ exibe um ótimo desempenho para a adsorção da maioria dos metais pesados, mas mostra uma afinidade maior por prata, mercúrio e ouro. Para o mesmo metal pesado, o $\mathrm{MoS}_{2}$ preparado por meio de diferentes métodos exibe desempenhos diferentes e isso pode ser explicado pelo fato de o mesmo possuir morfologia, espessura, sítios de absorção e oxidação diferentes (Ai et al., 2016a).

Por exemplo, nanofolhas de $\mathrm{MoS}_{2}$ sintetizadas por meio da esfoliação eletroquímica exibem uma ótima capacidade de adsorção de chumbo (Liu et al., 2017) muito maior que as nanofolhas de $\mathrm{MoS}_{2}$ sintetizadas pelo método hidrotérmico (Aghagoli et al., 2017). Quando o dissulfeto de molibdênio é modificado superficialmente ou estruturalmente, há uma maior capacidade de adsorção. A forma de gel permite que o $\mathrm{MoS}_{2}$ atinja uma capacidade de adsorção quase 40 vezes maior do que $\mathrm{o}$ $\mathrm{MoS}_{2}$ na forma de uma flor (Ma et al., 2018).

\subsubsection{Comparação de adsorventes à base de $\mathrm{MoS}_{2}$ com outros adsorventes}

Como dito anteriormente, o dissulfeto de molibdênio possui muitos sítios ativos que fornecem vários locais de adsorção de metais pesados. Embora a complexação seja considerada o principal efeito durante o remoção de metais pesados para a grande maioria dos adsorventes, aqueles sintetizados à base de enxofre exibem uma melhor complexação de metais pesados em comparação com outros grupos funcionais, o que permite com que os adsorventes baseados em $\mathrm{MoS}_{2}$ exibam um desempenho muito melhor do que outros adsorventes na remoção de íons de metais pesados da água e em efluentes.

$\mathrm{O} \mathrm{MoS}_{2}$ tem capacidade de adsorção muito maior do que outros adsorventes convencionais para a remoção de metais pesados. Como exemplo, nanofolhas de $\mathrm{MoS}_{2}$ atingem um nível de adsorção particularmente alto para a $\mathrm{Ag}^{+}$(cerca de 4000 $\mathrm{mg} / \mathrm{g}$ ) (Wang et al., 2018), que é bem maior do que vários adsorventes porosos (10-400 mg/g). 
Neste caso em específico, a remoção de $\mathrm{Ag}^{+}$por $\mathrm{MoS}_{2}$ é atribuída a maior afinidade entre os sítios ativos da superfície do enxofre e os íons $\mathrm{Ag}^{+}$em comparação com outros contaminantes. Ao ser aplicado para adsorver $\mathrm{Hg}^{2+}$, as nanofolhas $\mathrm{MoS}_{2}$ com maior espaçamento entre as camadas possuem capacidade máxima de adsorção de $2563 \mathrm{mg} / \mathrm{g}$ (Ai et al., 2016b) que é quase 14 vezes maior que o carvão ativado (Krishnan \& Anirudhan, 2002) e é também maior que compostos híbridos a base de EDTA.

\subsection{Mecanismo de adsorção}

Três mecanismos são utilizados para explicar a alta adsorção de metais pesados pelo dissulfeto de molibdênio: complexação, interação eletrostática, e reação redox. Devido à grande afinidade entre enxofre e metais pesados, os mesmos são adsorvidos pelos sítios livres de $\mathrm{S}$ expostos na superfície do $\mathrm{MoS}_{2}$ através da formação de complexos do tipo metal pesadoenxofre. Como exemplo, o íon $\mathrm{Hg}^{2+}$ pode se complexar com dois átomos de $\mathrm{S}$ quando a quantidade de $\mathrm{Hg}^{2+}$ é insuficiente para completar a reação de complexação (Ai et al., 2016b).

O mecanismo de complexação entre enxofre e um metal pesado é considerado um processo de adsorção primário. Devido a diferença de afinidade do enxofre por metais pesados, o $\mathrm{MoS}_{2}$ exibe capacidades de adsorção diferentes para os diversos tipos de metais pesados, o que pode ser melhorado caso ele esteja parcialmente oxidado (Liu et al., 2017).

$\mathrm{O}$ dissulfeto de molibdênio é carregado negativamente, o que facilita a atração de metais pesados carregados positivamente. Entretanto, a contribuição desta forma de adsorção tende a ficar menor quando se compara com a complexação química, visto que a superfície do $\mathrm{MoS}_{2}$ ficaria saturada por íons dos metais pesados reduzindo a eficiência de adsorção (Feng et al., 2018).

A interação eletrostática passa a ser um fator dominante com grande contribuição para metais pesados que não podem ligar $\mathrm{MoS}_{2}$ através da complexação, como é o caso do $\mathrm{Co}^{2+} \mathrm{e} \mathrm{Cu}^{2+}$ (Aghagoli et al., 2017). Outro detalhe é que a estequiometria está intimamente ligada ao processo de adsorção. Para o caso do $\mathrm{Hg}^{2+}$ a maior capacidade de adsorção é atingida para a proporção de 1:1 entre enxofre e mercúrio (Jia et al., 2017).

Quando o potencial redox dos pares redox de metal pesado é maior do que o do par $\mathrm{MoO}_{4}{ }^{2-}$ e $\mathrm{SO}_{4}{ }^{2-} / \mathrm{MoS}_{2}(0,429 \mathrm{~V})$, os metais pesados podem ser adsorvidos e reduzidos por $\mathrm{MoS}_{2}$ e nesse caso a reação redox é o mecanismo dominante para a remoção de metais pesados de solução aquosa. Outro detalhe é que foi observado que metais que apresentam maiores potenciais redox como $\mathrm{Ag}^{+}$e $\mathrm{Au}^{3+}$ são geralmente eliminados por este tipo de reação (Wang et al., 2016).

\subsection{Fatores que afetam a adsorção}

\subsection{1 pH da solução}

O potencial hidrogeniônico da solução possui papel fundamental no processo de adsorção, pois além de estabilizar os equilíbrios químicos presentes na solução também afeta as propriedades superficiais do $\mathrm{MoS}_{2}$. Como dito anteriormente, o dissulfeto de molibdênio possui carga negativa em todos os valores de $\mathrm{pH}$, o que indica que metais pesados serão atraídos eletrostaticamente em todos os valores de $\mathrm{pH}$. Como exemplo, o potencial zeta do $\mathrm{MoS}_{2} \mathrm{em} \mathrm{pH}=1$ é de cerca de $-1,0 \mathrm{mV}, \mathrm{o}$ que conduz a forças de atração muito fracas entre o material e os íons dos metais pesados (Jia et al., 2017).

Outro detalhe que deve ser levado em consideração e que de certa forma explica as baixas atrações e interações em pH baixos é o fato de que nessas faixas ocorre disputa entre os metais pesados e os íons hidrogênio e hidrônio pelos sítios ativos do $\mathrm{MoS}_{2}$, diminuindo, dessa forma, a capacidade de adsorção do mesmo (Wang et al., 2018). Para resolver este problema, é necessário fazer o processo de adsorção em pHs maiores, pois nessas faixas aumenta-se as cargas negativas do $\mathrm{MoS}_{2}$. 
Entretanto, esses valores de $\mathrm{pH}$ não podem ser demasiadamente altos pois nesse caso podem ocorrer reações químicas secundárias indesejadas entre os metais pesados e íons hidroxila presentes na solução podendo não serem adsorvidos na superfície do $\mathrm{MoS}_{2}$. Desta forma, a faixa de pH ideal para a adsorção de metais pesados está entre 5,0 e 8,0.

\subsubsection{Tempo de contato}

Assim como o pH, o tempo de contato é de grande importância para a adsorção de metais pesados por parte do dissulfeto de molibdênio. No início do processo a capacidade de adsorção é máxima e vai diminuindo com o passar do tempo pois a reação é levada para o equilíbrio químico (Wu et al., 2009). No início desse processo, a alta adsorção é um resultado direto da interação entre os sítios ativos do $\mathrm{MoS}_{2}$ e a concentração dos metais pesados em solução. Com a ocupação dos sítios ativos, este processo reduz a sua velocidade. É importante salientar que a capacidade de adsorção inicial está ligada à concentração inicial de metais pesados em solução e que, quanto maior a concentração desses metais, maior será a capacidade de adsorção do $\mathrm{MoS}_{2}$. A partir da saturação inicial dos sítios de adsorção do material, a reação se torna lenta e praticamente atinge o equilíbrio (Wang et al., 2018).

\subsubsection{Temperatura}

A temperatura também influencia diretamente no processo de adsorção de metais pesados pelo $\mathrm{MoS}_{2}$. Ela varia as interações entre os diferentes íons presentes em solução e o dissulfeto de molibdênio e sua elevação aumenta a capacidade de adsorção do $\mathrm{MoS}_{2}$. Essa capacidade é atribuída a uma grande complexação entre o $\mathrm{MoS}_{2} \mathrm{e}$ íons de metais pesados em solução, que por sua vez é atribuída a alta taxa de difusão de metais pesados para dentro da estrutura do dissulfeto de molibdênio (Peng et al., 2017).

Para entender como ocorre esse processo é importante entender como a reação ocorre em termos termodinâmicos avaliando parâmetros como entalpia $(\Delta \mathrm{H})$, entropia $(\Delta \mathrm{S})$ e energia livre de Gibbs $(\Delta \mathrm{G})$. Como exemplo, quando os íons $\mathrm{Co}^{2+} \mathrm{e}$ $\mathrm{Ni}^{2+}$ estão presentes em uma solução contendo $\mathrm{MoS}_{2}$, surge um valor negativo para $\Delta \mathrm{G}$, e valores positivos para $\Delta \mathrm{H}$ e $\Delta \mathrm{S}, \mathrm{o}$ que indica que estes processos são espontâneos, absorvem calor (endotérmicos) e são irreversíveis, o que o é extremamente importante, visto que uma vez absorvidos, esses íons não retornariam para a solução (Dong et al., 2019).

\subsubsection{Outros íons}

Os íons estranhos (íons diferentes dos metais pesados e que estão em solução) podem mudar significativamente a capacidade de adsorção de um adsorvente específico, uma vez que esses íons podem competir com os íons do metal pesado pelos sítios de adsorção do material, impedindo transferência de metais pesados da solução para a superfície do adsorvente (W. Peng et al., 2017).

Observa-se que não há influência de íons como $\mathrm{Na}^{1+}, \mathrm{Ca}^{2+}, \mathrm{Mg}^{2+}$. Como exemplo, a concentração de mercúrio II nas águas residuais da indústria pode ser reduzida de $126 \mu \mathrm{g} / \mathrm{L}$ para $0,055 \mu \mathrm{g} / \mathrm{L}$ após a purificação com $\mathrm{MoS}_{2}$, enquanto a concentração de outros íons como $\mathrm{Na}^{1+}, \mathrm{K}^{1+}, \mathrm{Ca}^{2+}, \mathrm{Mg}^{2+}, \mathrm{Al}^{3+}$, etc., nas mesmas águas residuais não varia significativamente (Ai et al., 2016a).

A alta seletividade do dissulfeto de molibdênio a diferentes tipos de metais pesados e resistência a íons estranhos pode estar relacionada a interações intermoleculares específicas entre o $\mathrm{MoS}_{2}$ e metais pesados. Deve ser apontado que $\mathrm{MoS}_{2}$ exibe diferentes capacidades de adsorção para diferentes metais pesados quando eles existem simultaneamente na solução e a capacidade de adsorção depende em grande parte da maciez dos metais pesados (Pearson, 1968). 


\subsection{Compósitos à base de $\mathrm{MoS}_{2}$ como catalisadores de fotodegradação}

$\mathrm{O} \mathrm{MoS}_{2}$ pode ser associado a diversos tipos de materiais, incluindo óxidos, nitretos, metais alcalinos, matais de transição entre outros para aumento da quantidade de metais adsorvidos em sua estrutura. Ele pode se ligar ao grafeno pela rota solvotérmica, um material que já possui muitas propriedades conhecidas.

As boas propriedades elétricas fazem com que o grafeno atue como um excelente aceitador/transportador de elétrons o que aumenta significativamente a adsorção de poluentes. A incorporação do $\mathrm{MoS}_{2}$ ao grafeno apresenta um bom desempenho como catalisador, uma vez que $\mathrm{MoS}_{2}$ disperso na superfície do grafeno aproveita ao máximo as propriedades do grafeno. O $\mathrm{MoS}_{2} /$ óxido de grafeno sintetizado em DMF pode recozido para melhorar a cristalinidade do material formado (Yuan et al., 2017).

As amostras preparadas desta forma têm uma alta concentração de $\mathrm{MoS}_{2}$ nas bordas do material e possuíam três camadas. Nesse caso, uma solução de azul de metileno com concentração de $15 \mathrm{mg} / \mathrm{L}$ foi utilizada para avaliar a eficiência fotocatalítica do material sintetizado (Yuan et al., 2017).

Neste teste, uma amostra de $10 \mathrm{mg}$ do catalisador foi colocada em uma solução com volume de $50 \mathrm{~mL}$ de azul de metileno e os resultados sugeriram que o ele poderia ser totalmente degradado pelo compósito formado por MoS $2 / 0$ xido de grafeno através da radiação UV e da luz visível com processos catalíticos que levaram 50 minutos e 75 minutos respectivamente. A combinação destes dois materiais leva a melhoria do desempenho de fotodegradação (Li et al., 2014).

Compósitos de $\mathrm{MoS}_{2} /$ óxido de grafeno podem também ser preparados pelo método assistido por micro-ondas (Pan et al., 2013). Através desta técnica, folhas de $\mathrm{MoS}_{2}$ foram observadas entrelaçadas nas folhas onduladas de óxido de grafeno. As superfícies estão conectadas de forma estável e isso contribui para a adsorção dos poluentes. O material preparado por este método atingiu uma taxa de degradação do azul de metileno em torno de $99 \%$ em 60 min sob a luz visível.

Outro método que também pode ser utilizado na produção de híbridos de $\mathrm{MoS}_{2} /$ óxido de grafeno é a sonicação, que produz compósitos com morfologia mais complexa e heterogênea (Cravanzola et al., 2016). O MoS 2 acoplado ao óxido de grafeno pode ainda ser inserido em outros materiais semicondutores formando outros tipos de catalisadores. O compósito dopado com $1 \%$ de zinco alcançou maior desempenho de fotodegradação. Uma solução com $50 \mathrm{~mL}$ de azul de metileno com concentração igual a 15,9 mg/L foi degradada em 98\% em 60 min sob a luz solar natural (Kumar et al., 2016).

Além disso, a mesma concentração de carbendazim também foi degradada em $97 \%$ nas mesmas condições. O motivo para sua alta eficiência catalítica é a capacidade de transferir elétrons de forma rápida através do material, o que produz radicais livres · $\mathrm{OH}$ altamente reativos e que tem uma maior capacidade de degradação de contaminantes (Kumar et al., 2016). A partir disso pode-se concluir que a intercalação do $\mathrm{MoS}_{2}$ ao óxido de grafeno agrega as propriedades dos dois materiais, principalmente pelo fato da boa condutividade elétrica das folhas de grafeno inibirem a recombinação de espécies já degradadas na solução aquosa.

O nitreto de carbono puro possui baixa absorção de luz solar (Liao et al., 2012) e seu acoplamento com o dissulfeto de molibdênio pode aumentar sua capacidade de absorção (Peng \& Li, 2014).

As estruturas formadas entre as nanofolhas $\mathrm{MoS}_{2}$ (com cerca de $3 \%$ em massa) e as nanofolhas $\mathrm{C}_{3} \mathrm{~N}_{4}$ possuem uma área superficial muito grande e isso ajuda muito no processo de degradação de contaminantes, pois aumenta os sítios de adsorção (Tisseraud et al., 2016). Com o intuito de avaliar a atividade fotocatalítica do compósito, o mesmo foi testado com uma solução de efluente orgânico simulado e obteve uma taxa de degradação de $92,4 \%$ depois de ser irradiado com luz visível por 2 horas (Lu et al., 2016).

Ao comparar a eficiência de remoção do $\mathrm{C}_{3} \mathrm{~N}_{4}$ puro com sua associação ao $\mathrm{MoS}_{2}$ percebe-se uma notável diferença de adsorção devido as camadas estruturais formadas entre as folhas ultrafinas $\mathrm{MoS}_{2}$ entrelaçadas a estrutura do $\mathrm{C}_{3} \mathrm{~N}_{4}$. Dessa forma, conclui-se que a intercalação dos dois materiais produz mais sítios ativos na estrutura que a adsorção se deve às 
nanofolhas de $\mathrm{MoS}_{2}$. Entretanto, a sobreposição das camadas de dissulfeto de molibdênio sobre as camadas de nitreto de carbono diminui a taxa de degradação de poluentes por parte do material, por isso, é importante controlar sua quantidade na estrutura do material (Lu et al., 2016).

O dissulfeto de molibdênio pode também ser associado ao óxido de titânio principalmente devido as suas várias propriedades, como estabilidade química, baixa toxicidade e baixo custo (Dong et al., 2015). Entretanto, um fator limitante é o fato do $\mathrm{TiO}_{2}$ absorver na região do UV (Zhong \& Li, 2012).

Para resolver este problema e consequentemente aumentar a capacidade de absorção do compósito, pode-se sintetizálos em conjunto usando a técnica da eletrofiação. Nesta técnica, prepara-se o $\mathrm{MoS}_{2}$ pelo método hidrotérmico e em seguida o mesmo é eletrofiado sobre o $\mathrm{TiO}_{2}$ para formar uma estrutura de rede 3D (Zhang et al., 2016).

A eficiência catalítica dessa associação foi avaliada para absorção de corantes orgânicos e também com solução de matéria orgânica simulada e a taxa de degradação do corante foi de mais de $95 \%$ para o corante e para a matéria orgânica simulada com irradiação de luz UV (Jia et al., 2014).

Microcápsulas de $\mathrm{TiO}_{2}$ tem uma boa atividade catalítica devido à sua grande área superficial quando se compara o mesmo composto sintetizado na forma de nanofibras (Qi et al., 2014). Para tanto, nanofolhas de dissulfeto de molibdênio podem ser preparadas pelo método hidrotérmico para revestir nanoesferas de óxido de titânio (Low et al., 2017) e quando se compara a capacidade de adsorção do $\mathrm{MoS}_{2}$ ou $\mathrm{TiO}_{2}$ puros com o compósito formado pela junção destes dois materiais observa-se uma maior capacidade de adsorção.

Com o intuito de melhorar a atividade catalítica do $\mathrm{TiO}_{2}$, antes do acoplá-lo ao $\mathrm{MoS}_{2}$ é interessante dopar este composto com impurezas para expandir sua de adsorção para a luz visível. Dessa forma, o óxido de titânio modificado e intercalado ao dissulfeto de molibdênio irá melhorar o desempenho de degradação dos poluentes orgânicos e inorgânicos ( Liu et al., 2017).

$\mathrm{O} \mathrm{TiO}_{2}$ dopado com $\mathrm{N}$ e estes associados ao $\mathrm{MoS}_{2}$ foram responsáveis pela remoção de mais de $91 \%$ de contaminantes baseados em matéria orgânica artificial, com concentração de $10 \mathrm{mg} / \mathrm{L}$ e recebendo luz visível por 2 horas quando comparado apenas com o $\mathrm{TiO}_{2}$ puro (Liu et al., 2017).

Catalisadores tendo como base compostos do bismuto são muito interessantes pois o mesmo possui boas propriedades ópticas e o oxibometo de bismuto, em especial, tem desempenho fotocatalítico maior que os outros derivados do bismuto. Com o intuito de melhorar o desempenho do $\mathrm{BiOBr}$, o mesmo pode ser associado ao $\mathrm{MoS}_{2}$ pelo método solvotérmico (Di et al., 2014).

Cerca de 0,02 g dessa associação (com 3\% em massa de $\mathrm{MoS}_{2}$ ) foi usada em uma solução de rodamina com concentração de $10 \mathrm{mg} / \mathrm{L}$ recebendo luz visível e houve uma grande degradação do corante. Isso se deve ao fato da superfície porosa do compósito $\mathrm{MoS}_{2} / \mathrm{BiOBr}$ fornecer mais sítios ativos que ajudam na degradação das rodaminas e essas características são potencializadas pelas camadas finas de nanofolhas $\mathrm{MoS}_{2}$ e pelas bandas de energia do BiOBr.

Um outro material que pode ser acoplado ao $\mathrm{MoS}_{2}$ é o BiOI, pois possui uma melhor resposta a luz visível mas um problema que pode atrapalhar sua utilização e a lentidão no processo de separação de fotoelétrons e a solução seria liga-lo ao $\mathrm{MOS}_{2}$ para melhorar suas propriedades e consequentemente aumentar sua resposta e as interações com a luz visível.

$\mathrm{O}$ carbonato de bismuto, $\mathrm{Bi}_{2} \mathrm{O}_{2} \mathrm{CO}_{3}$ também pode ser associado ao $\mathrm{MoS}_{2}$, o problema reside no fato de que o $\mathrm{Bi}_{2} \mathrm{O}_{2} \mathrm{CO}_{3}$ tem um desempenho catalítico ativado pela luz UV (Ni et al., 2016). Nessa junção, usa-se o método hidrotérmico para a síntese da associação de $\mathrm{Bi}_{2} \mathrm{O}_{2} \mathrm{CO}_{3} / \mathrm{MoS}_{2}$ (Wang et al., 2014).

Ao colocar 0,5\% em massa de $\mathrm{MoS}_{2}$ em $\mathrm{Bi}_{2} \mathrm{O}_{2} \mathrm{CO}_{3}$, o material sintetizado mostrou um desempenho fotocatalítico melhorado, mais de $99 \%$ das rodaminas com concentração de $10 \mathrm{mg} / \mathrm{L}$ e volume total de $50 \mathrm{~mL}$ foi removido em $2,5 \mathrm{~h}$ recebendo luz UV. 
Em outro estudo, nanobastões $\mathrm{Bi}_{2} \mathrm{~S}_{3}$ foram incorporados a estrutura do $\mathrm{MoS}_{2}$ pelo método hidrotérmico e o efeito catalítico do compósito foi medido utilizando vermelho de fenol em solução e quando a relação em massa entre $\mathrm{Bi}_{2} \mathrm{~S}_{3}$ e $\mathrm{MoS}_{2}$ foi de 4:1, o compósito exibiu grande eficiência de remoção de poluentes ao receber irradiação de luz visível (Vattikuti \& Byon, 2016).

A intercalação de $\mathrm{MoS}_{2}$ na estrutura do $\mathrm{Bi}_{2} \mathrm{~S}_{3}$ aumenta a área de superfície e sites ativos de compostos, que forneceram mais sítios para elétrons fotoinduzidos e lacunas para participar da reação redox, resultando em maior atividade fotocatalítica (Long et al., 2016). Esse material foi capaz de reduzir contaminantes do tipo atrazina. Nesse teste, 30 mg do fotocatalisador composto foi adicionado a $60 \mathrm{~mL}$ de uma solução de atrazina com concentração de $5 \mathrm{mg} / \mathrm{L}$ e o compósito apresentou boa atividade catalítica eliminando cerca 89\% da atrazina em 240 min de reação fotocalítica (Long et al., 2016).

O dissulfeto de molibdênio pode ainda ser associado a prata em processos fotocalíticos, tendo como precursores iniciais o fosfato e o nitrato de prata. A prata já está presente em vários semicondutores e principal problema reside no fato da prata sofrer foto-corrosão durante o processo fotocatalítico (Wang et al., 2012).

Dessa forma, é extremamente importante associar a prata a outros materiais. Wang et. al. associaram o $\mathrm{MoS}_{2}$ ao $\mathrm{Ag}_{3} \mathrm{PO}_{4}$ pelo método da deposição e percebeu que as nanopartículas de fosfato de prata estavam uniformemente dispersas na superfície das nanofolhas $\mathrm{MoS}_{2} \mathrm{e}$ ambos os componentes estavam em contato próximo um com o outro.

Outro estudo conduzido por Zhu et al., mostrou que com 0,648\% em massa de $\mathrm{MoS}_{2}$ ocorreu o melhor desempenho catalítico da associação, com excelentes propriedades de degradação fotocatalítica para azul de metileno, rodaminas, matéria orgânica simulada e compostos fenólicos, que foram quase completamente degradados (Zhu et al., 2016). Esse material era excitado de forma mais fácil pela luz visível para produzir fotoelétrons, o que melhora muito o processo de fotocatálise.

Com o intuito de aumentar a capacidade catalítica e a estabilidade, Wan et al. usou vários métodos de síntese, entre eles o método hidrotérmico, esfoliação ultrassônica, crescimento in situ, para sintetizar a nanopartículas de $\mathrm{Ag}_{3} \mathrm{PO}_{4}$ associadas a $\mathrm{MoS}_{2}$ com poucas camadas (Wan et al., 2017).

No caso da esfoliação ultrassônica ele utilizou uma pequena quantidade de $\mathrm{MoS}_{2}$ que foi disperso em N-metil-2pirrolidona (NMP), seguido por esfoliação ultrassônica por 10 h para obtenção de suspensão. Depois disso, as nanopartículas de nitrato de prata foram misturadas ao dissulfeto de molibdênio preparado em solução para obter o fotocatalisador e após 16 minutos de exposição a luz visível formou-se as nanopartículas.

Este fotocatalisador foi testado frente uma solução de rodaminas (com concentração de $10 \mathrm{mg} / \mathrm{L}$ ) e verificou-se uma alta eficiência do mesmo, degradando-as com apenas $6 \mathrm{~mL}$ de solução (Wan et al., 2017).

Já foi relatado anteriormente sobre a associação entre $\mathrm{MoS}_{2}$ e $\mathrm{TiO}_{2}$. Além dessa, há outras associação entre esses materiais que são promissoras, entre elas destaca-se a intercalação de dissulfeto de molibdênio com óxido de zinco, que é um semicondutor muito utilizado em diversas pesquisas, contudo, ele possui uma pequena faixa de absorção na região visível do espectro solar (Kolodziejczak-Radzimska \& Jesionowski, 2014).

A performance catalítica do $\mathrm{ZnO}$ pode ser melhorada pela associação com outros semicondutores semelhantes (Zheng et al., 2017). Com base nisso, composições entre $\mathrm{MoS}_{2} / \mathrm{ZnO}$ foram produzidas pelo método hidrotérmico em baixa temperatura (Tan et al., 2014).

Os catalisadores preparados $(10 \mathrm{~mL})$ foram dispersos em azul de metileno (com concentração igual a $20 \mathrm{mg} / \mathrm{L}$ ) e simulou-se a luz solar com uma lâmpada de $18 \mathrm{~W}$. A irradiação foi realizada por 100 min e após esse tempo os autores perceberam que houve uma redução de $92,7 \%$ do teor de azul de metileno em solução.

Já Awasthi et al. relataram que os compósitos $\mathrm{MoS}_{2} / \mathrm{ZnO}$ sintetizados mostraram uma alta atividade fotocatalítica em solução de vermelho de fenol. Sob irradiação UV, aproximadamente $93 \%$ do vermelho de fenol foi degradado dentro 50 min. 
Enquanto isso, $90 \%$ do vermelho de fenol pode ser removido dentro 80 min sob a iluminação de luz solar natural (Awasthi et al., 2016).

Pode também associar dissulfeto de molibdênio a óxido cúprico, tendo em vista a sua boa atividade catalítica (Arai et al., 2008). A fotodegradabilidade da associação $\mathrm{MoS}_{2} / \mathrm{CuO}$ foram testadas em uma solução de azul de metileno sob luz UVVis. Depois de irradiado por 100 min, a quantidade de azul de metileno removida foi cerca de 95,7\%.

O dióxido de zircônio é um material emergente que pode ser utilizado associado ao dissulfeto de molibdênio. Entre suas características, a maior desvantagem é a pequena resposta a energia solar (apenas 4\%) e isso constitui a principal dificuldade em sua utilização prática (Hao et al., 2017).

Com o intuito de melhorar a resposta óptica do $\mathrm{ZrO}_{2}$ os autores fizeram algumas associações como formação de compósitos, dopagem de semicondutores e deposição de íons metálicos na estrutura do material (Renuka et al., 2017). Junções de $\mathrm{ZrO}_{2} / \mathrm{MoS}_{2}$ foram testadas em soluções de matéria orgânica simulada e a amostra preparada, com teor de $20 \%$ em massa de $\mathrm{MoS}_{2}$ exibiu a maior eficiência de fotodegradação sob luz ultravioleta com uma eficiência de degradação de cerca de $95,2 \%$ (Sun et al., 2017). Isso se deve basicamente ao efeito da combinação das diferentes estruturas dos dois materiais, alta área de superficial, vários sítios ativos e alta porosidade do dissulfeto de molibdênio.

Vários óxidos de molibdênio do tipo MoOx também podem ser combinados com $\mathrm{MoS}_{2}$ para serem utilizados na remoção de poluentes ambientais (Cummins et al., 2015). Zhou et al. prepararam estruturas compostas por $\mathrm{MoS}_{2} / \mathrm{MoOx}$ alinhadas verticalmente pelo método da deposição de vapor e o teste de desempenho fotocatalítico foi realizado degradando 8 $\mathrm{mL}$ de solução aquosa de rodaminas com concentração igual a $4 \times 10^{-6} \mathrm{~mol} / \mathrm{L}$ sendo que a maior taxa de degradação chegou a 97\% após 120 min de irradiação de luz visível. (Zhou et al., 2014).

Uma outra associação possível é sintetizar compostos ternários tendo o dissulfeto de molibdênio como um dos constituintes. Como exemplo, o compósito formado pela junção do tipo $\mathrm{Fe}_{3} \mathrm{O}_{4} / \mathrm{MoS}_{2} / \mathrm{Ag}_{3} \mathrm{PO}_{4}$ melhorou o desempenho fotocatalítico para corantes orgânicos sob irradiação de luz visível em comparação com o material individual (Guo et al.,

2016). Essa associação, recebendo radiação de luz visível, foi capaz de eliminar até $98,90 \%$ de uma solução de rodaminas com concentração de $20 \mathrm{mg} / \mathrm{L}$ e cerca de $90,74 \%$ de uma solução de vermelho de fenol com concentração igual a 30 mg/L após 10 minutos de irradiação. Os autores relatam ainda que os íons $\mathrm{Fe}^{2+} \mathrm{e} \mathrm{Fe}^{3+}$ possuem fundamental importância nesse processo pois desempenham o papel de "armadilhas" de elétrons, o que facilita o processo de oxidação de contaminantes. Outro detalhe é que a nanofolhas de $\mathrm{MoS}_{2}$ fornecem uma área superficial adicional na superfície do material.

Diante disso, a incorporação de óxido de metais de transição em nanopartículas de $\mathrm{MoS}_{2}$ podem promover fotodegradação, sendo atribuído à redução da taxa de recombinação de fotogerados elétrons e buracos. Além disso, as nanofolhas $\mathrm{MoS}_{2}$ fornecem uma alta área de superfície específica, o que permite que mais metais sejam deposita-se na estrutura lamelar e contribui para a formação de estruturas (Guo et al., 2016).

A Tabela 2 mostra as diferentes formas de associações do $\mathrm{MoS}_{2}$ com outros materiais, que incluem derivados do zinco, carbono, titânio, carbetos, ferro, fibras, resinas e óxidos juntamente com a forma física, métodos de síntese, contaminantes que conseguem reter, $\mathrm{pH}$ de eficiência, tempo de reação e porcentagem de eficiência. 
Tabela 2: Resumo da aplicação de algumas associações do dissulfeto de molibdênio.

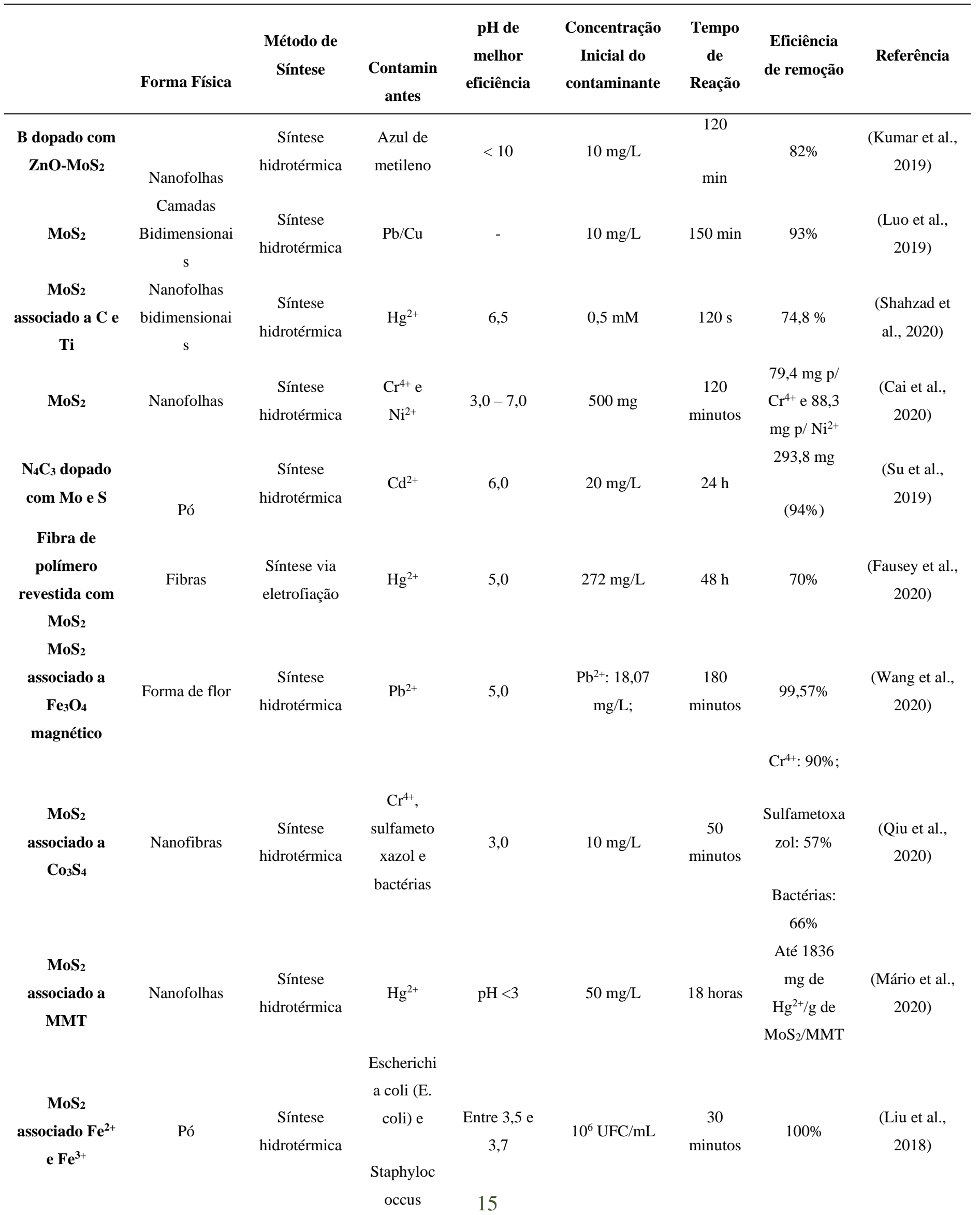




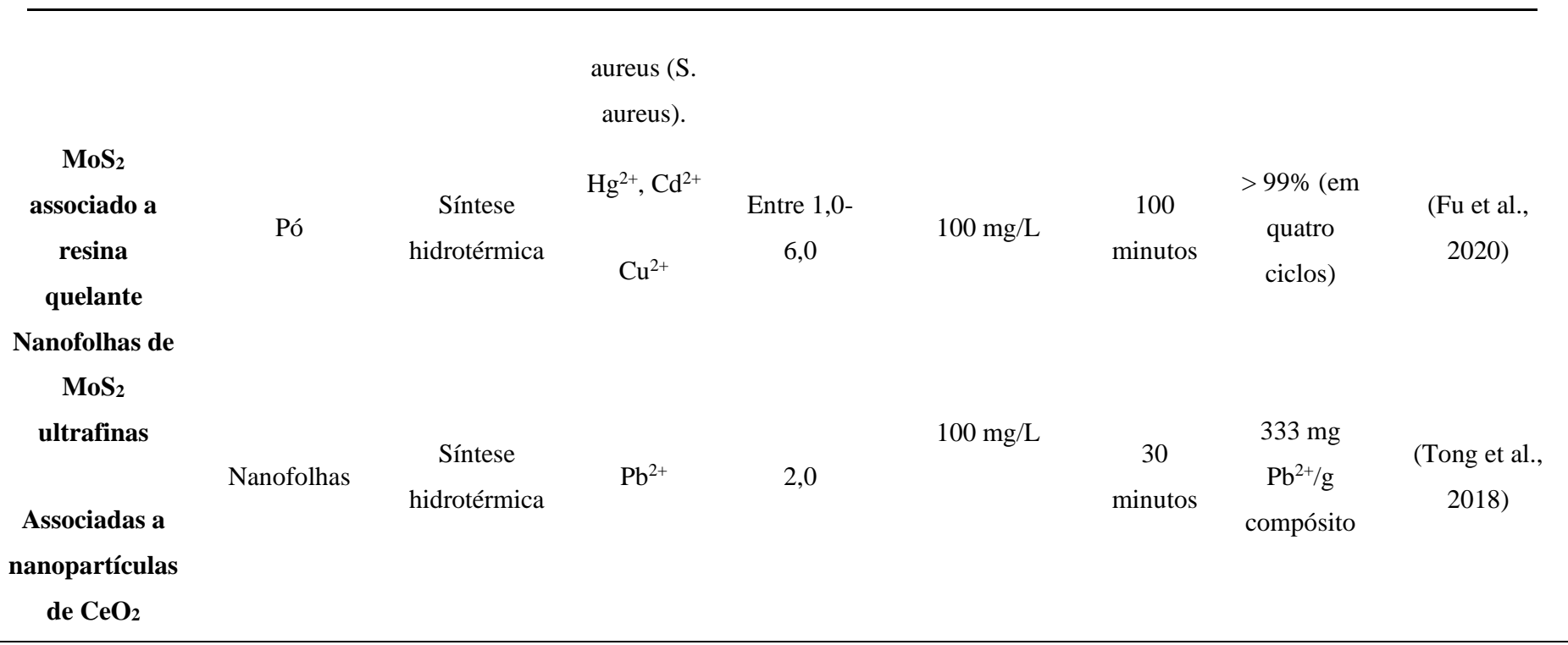

Fonte: Autores (2021).

De acordo com os dados da Tabela 2, é possível observar que o dissulfeto de molibdênio pode ser associado a diferentes materiais, se apresenta a em formas físicas diversas, possui diferentes métodos de síntese, pode reter vários tipos de contaminantes, inclusive bactérias do tipo Escherichia coli e Staphylococcus aureus.

Outro detalhe de sua aplicação se refere ao pH de utilização, sendo que há predominância de pHs ácidos para uma maior eficiência de remoção.

\section{Conclusão}

O dissulfeto de molibdênio é um material amplamente utilizado na remediação ambiental, pois exibe uma taxa de remoção de poluentes extremamente alta, rápida cinética de adsorção, boa afinidade e excelente seletividade para adsorver metais pesados e vários íons na água. O método de síntese que apresentou melhor desempenho em termos de rendimento foi o método hidrotérmico e este é também o método mais utilizado para a síntese do $\mathrm{MoS}_{2}$. O dissulfeto de molibdênio pode ainda ser combinado com diversos outros materiais, como óxido de titânio, grafeno, nitretos de carbono, compostos derivados da prata, bismuto, entre outros. Como exemplo, nanofolhas de $\mathrm{MoS}_{2}$ atingem um nível de adsorção particularmente alto para a $\mathrm{Ag}+$ (cerca de $4000 \mathrm{mg} / \mathrm{g}$ ), que é bem maior do que vários adsorventes porosos (10-400 mg/g). Outro exemplo é a associação de $\mathrm{MoS}_{2} / \mathrm{ZrO}_{2}$ (contendo $20 \%$ em massa de $\mathrm{MoS}_{2}$ ), que exibiu uma eficiência de fotodegradação sob luz ultravioleta em torno de 95,2\% quando usada em matéria orgânica simulada. A associação $\mathrm{MoS}_{2}$ com óxidos a base de molibdênio também produz bons resultados. $\mathrm{O}$ teste de desempenho utilizando $8 \mathrm{~mL}$ de rodaminas com concentração igual a $0,4 \times 10^{-5} \mathrm{~mol} / \mathrm{L}$ frente a esta associação resultou em uma taxa de degradação de 97\% após 120 minutos de irradiação de luz visível. No teste com águas residuais e considerando pHs entre 2 e 7, a eficiência de degradação ficou em 70\%. Outro exemplo é sua associação com compostos derivados do ferro e prata (como $\mathrm{Fe}_{3} \mathrm{O}_{4}$ e $\mathrm{Ag}_{3} \mathrm{PO}_{4}$ ), em que os mesmos foram testados frente ao contaminante vermelho de fenol com concentração igual a $30 \mathrm{mg} / \mathrm{L}$, sendo que a associação degradou este contaminante em $98 \%$ em cerca de 10 minutos de atividade. Essas associações melhoram a eficiência de remoção de diferentes poluentes orgânicos e inorgânicos, fato que foi evidenciado nos testes com soluções de rodaminas, azul de metileno, fenóis e matéria orgânica simulada através de mecanismos como complexação química, interação eletrostática e reação redox. É extremamente importante criar maneiras de controlar a quantidade de camadas do $\mathrm{MoS}_{2}$ afim de obter uma maior área superficial do material e consequentemente aumentar a eficiência de adsorção do mesmo. Alguns fatores tem grande importância no processo de 
adsorção, como a estrutura do $\mathrm{MoS}_{2}$, tempo de adsorção, tempo de uso e outros estão relacionadas as condições da solução, como $\mathrm{pH}$, temperatura e tipos de íons presentes. Esses parâmetros possuem um efeito significativo no processo de adsorção. Embora o $\mathrm{MoS}_{2}$ tenha apresentado bons resultados na remediação ambiental, alguns desafios ainda precisam ser vencidos antes da aplicação em grande escala. Entre esses problemas incluem baixa taxa de recuperação, a rápida separação do mesmo da solução em que está inserido aliados ao alto custo de fabricação e o complicado processo de produção, que podem limitar sua aplicação prática.

Embora grandes esforços tenham sido feitos na exploração de $\mathrm{MoS}_{2}$ para a aplicação na remoção de metais pesados, algumas questões ainda permanecem abertas e muitos desafios ainda precisam ser superados antes de sua aplicação na prática e de forma comercial. Alguns desses problemas são a sua separação rápida da solução em que está inserido, alto custo de fabricação e complicado processo de produção. Além disso, é necessário aumentar a produção do MoS 2 , reduzir o custo, facilitar a operação e dar mais atenção à melhoria de sítios ativos de grupos sulfonados durante o processo de síntese. Aliado a isso, ainda existem muitos problemas com a degradação de poluentes reais em compósitos à base de $\mathrm{MoS}_{2}$, principalmente a falta de conhecimento de todos os mecanismos que ocorrem nesse tipo de reação química. Com a resolução destes problemas espera-se que nanocompósitos de $\mathrm{MoS}_{2}$ sejam fotocatalisadores promissores no campo de fotodegradação de poluentes orgânicos, o que associado a compósitos ecologicamente favoráveis associe melhor custo de produção ao compromisso ambiental de produzir menos contaminação e menos lixo para remediar a ação antrópica, trazendo plenitude ao conceito da economia circular aplicada à ciência.

\section{Agradecimentos}

Agradecemos à CAPES, ao CNPq e à FACEPE pelo apoio ao desenvolvimento de pesquisa no LEIMO-IPCM/ Univasf.

\section{Referências}

Aghagoli, M. J., Hossein Beyki, M., \& Shemirani, F. (2017). Application of dahlia-like molybdenum disulfide nanosheets for solid phase extraction of Co(II) in vegetable and water samples. Food Chemistry, 223, 8-15. https://doi.org/10.1016/j.foodchem.2016.12.023

Ahn, C., Lee, J., Kim, H. U., Bark, H., Jeon, M., Ryu, G. H., Lee, Z., Yeom, G. Y., Kim, K., Jung, J., Kim, Y., Lee, C., \& Kim, T. (2015). Low-Temperature Synthesis of Large-Scale Molybdenum Disulfide Thin Films Directly on a Plastic Substrate Using Plasma-Enhanced Chemical Vapor Deposition. Advanced Materials, 27(35), 5223-5229. https://doi.org/10.1002/adma.201501678

Ai, K., Ruan, C., Shen, M., \& Lu, L. (2016a). MoS2 Nanosheets with Widened Interlayer Spacing for High-Efficiency Removal of Mercury in Aquatic Systems. Advanced Functional Materials, 26(30), 5542-5549. https://doi.org/10.1002/adfm.201601338

Ai, K., Ruan, C., Shen, M., \& Lu, L. (2016b). MoS2 Nanosheets with Widened Interlayer Spacing for High-Efficiency Removal of Mercury in Aquatic Systems. Advanced Functional Materials, 26(30), 5542-5549. https://doi.org/10.1002/adfm.201601338

Akple, M. S., Low, J., Liu, S., Cheng, B., Yu, J., \& Ho, W. (2016). Fabrication and enhanced CO2 reduction performance of N-self-doped TiO2 microsheet photocatalyst by bi-cocatalyst modification. Journal of CO2 Utilization, 16, 442-449. https://doi.org/10.1016/j.jcou.2016.10.009

Anoop Krishnan, K., \& Anirudhan, T. S. (2002). Removal of mercury(II) from aqueous solutions and chlor-alkali industry effluent by steam activated and sulphurised activated carbons prepared from bagasse pith: Kinetics and equilibrium studies. Journal of Hazardous Materials, 92(2), 161-183. https://doi.org/10.1016/S0304-3894(02)00014-6

Arai, T., Yanagida, M., Konishi, Y., Iwasaki, Y., Sugihara, H., \& Sayama, K. (2008). Promotion effect of CuO co-catalyst on WO3-catalyzed photodegradation of organic substances. Catalysis Communications, 9(6), 1254-1258. https://doi.org/10.1016/j.catcom.2007.11.012

Awasthi, G. P., Adhikari, S. P., Ko, S., Kim, H. J., Park, C. H., \& Kim, C. S. (2016). Facile synthesis of ZnO flowers modified graphene like MoS2 sheets for enhanced visible-light-driven photocatalytic activity and antibacterial properties. Journal of Alloys and Compounds, 682, 208-215. https://doi.org/10.1016/j.jallcom.2016.04.267

Cai, W., Dionysiou, D. D., Fu, F., \& Tang, B. (2020). CTAB-intercalated molybdenum disulfide nanosheets for enhanced simultaneous removal of Cr(VI) and $\mathrm{Ni}(\mathrm{II})$ from aqueous solutions. Journal of Hazardous Materials, 396(January), 122728. https://doi.org/10.1016/j.jhazmat.2020.122728

Castro, S., Lopez-Valdivieso, A., \& Laskowski, J. S. (2016). Review of the flotation of molybdenite. Part I: Surface properties and floatability. International Journal of Mineral Processing, 148, 48-58. https://doi.org/10.1016/j.minpro.2016.01.003 
Chabot, V., Higgins, D., Yu, A., Xiao, X., Chen, Z., \& Zhang, J. (2014). A review of graphene and graphene oxide sponge: Material synthesis and applications to energy and the environment. Energy and Environmental Science, 7(5), 1564-1596. https://doi.org/10.1039/c3ee43385d

Chang, K., \& Chen, W. (2011). In situ synthesis of MoS2/graphene nanosheet composites with extraordinarily high electrochemical performance for lithium ion batteries. Chemical Communications, 47(14), 4252-4254. https://doi.org/10.1039/c1cc10631g

Chen, S., Hu, Y., Meng, S., \& Fu, X. (2014). Study on the separation mechanisms of photogenerated electrons and holes for composite photocatalysts gC3N4-WO3. Applied Catalysis B: Environmental, 150-151, 564-573. https://doi.org/10.1016/j.apcatb.2013.12.053

Coleman, J. N., Lotya, M., O’Neill, A., Bergin, S. D., King, P. J., Khan, U., Young, K., Gaucher, A., De, S., Smith, R. J., Shvets, I. V., Arora, S. K., Stanton, G., Kim, H. Y., Lee, K., Kim, G. T., Duesberg, G. S., Hallam, T., Boland, J. J., ... Nicolosi, V. (2011). Two-dimensional nanosheets produced by liquid exfoliation of layered materials. Science, 331(6017), 568-571. https://doi.org/10.1126/science.1194975

Cravanzola, S., Cesano, F., Magnacca, G., Zecchina, A., \& Scarano, D. (2016). Designing rGO/MoS2 hybrid nanostructures for photocatalytic applications. RSC Advances, 6(64), 59001-59008. https://doi.org/10.1039/c6ra08633k

Cuellar, E. L., Martínez-De La Cruz, A., Torres, N. C., \& Cortez, J. O. (2015). Deposition of BiOBr thin films by thermal evaporation and evaluation of its photocatalytic activity. Catalysis Today, 252, 2-6. https://doi.org/10.1016/j.cattod.2015.01.013

Cui, L., Wang, Y., Gao, L., Hu, L., Yan, L., Wei, Q., \& Du, B. (2015). EDTA functionalized magnetic graphene oxide for removal of Pb(II), Hg(II) and Cu(II) in water treatment: Adsorption mechanism and separation property. Chemical Engineering Journal, 281, 1-10. https://doi.org/10.1016/j.cej.2015.06.043

Cummins, D. R., Martinez, U., Kappera, R., Voiry, D., Martinez-garcia, A., Jasinski, B., Kelly, D., Chhowalla, M., Mohite, A. D., Sunkara, M. K., \& Gupta, G. (2015). Catalytic Activity in Lithium Treated Core-Shell MoO x/MoS 2 Nanowires.

Di, J., Xia, J., Ge, Y., Xu, L., Xu, H., Chen, J., He, M., \& Li, H. (2014). Facile fabrication and enhanced visible light photocatalytic activity of few-layer MoS2 coupled BiOBr microspheres. Dalton Transactions, 43(41), 15429-15438. https://doi.org/10.1039/c4dt01652a

Dong, H., Zeng, G., Tang, L., Fan, C., Zhang, C., He, X., \& He, Y. (2015). An overview on limitations of TiO2-based particles for photocatalytic degradation of organic pollutants and the corresponding countermeasures. Water Research, 79, 128-146. https://doi.org/10.1016/j.watres.2015.04.038

Dong, L., Li, Q., Liao, Q., Sun, C., Li, X., Zhao, Q., Shen, R., Zhao, B., Asiri, A. M., Marwani, H. M., Wu, X., \& Hu, B. (2019). Characterization of molybdenum disulfide nanomaterial and its excellent sorption abilities for two heavy metals in aqueous media. Separation Science and Technology (Philadelphia), 54(6), 847-859. https://doi.org/10.1080/01496395.2018.1515226

Eda, G., Yamaguchi, H., Voiry, D., Fujita, T., Chen, M., \& Chhowalla, M. (2011). N1201874W.Pdf. Nano Letters, 5111-5116. https://doi.org/10.1021/n1201874w

Fausey, C. L., Zucker, I., Lee, D. E., Shaulsky, E., Zimmerman, J. B., \& Elimelech, M. (2020). Tunable Molybdenum Disulfide-Enabled Fiber Mats for HighEfficiency Removal of Mercury from Water. ACS Applied Materials \& Interfaces, 12(16), 18446-18456. https://doi.org/10.1021/acsami.9b22823

Feng, B., Yao, C., Chen, S., Luo, R., Liu, S., \& Tong, S. (2018). Highly efficient and selective recovery of Au(III) from a complex system by molybdenum disulfide nanoflakes. Chemical Engineering Journal, 350(Iii), 692-702. https://doi.org/10.1016/j.cej.2018.05.130

Fu, W., Ji, G., Chen, H., Yang, S., Guo, B., Yang, H., \& Huang, Z. (2020). Molybdenum sulphide modified chelating resin for toxic metal adsorption from acid mine wastewater. Separation and Purification Technology, 251(February), 117407. https://doi.org/10.1016/j.seppur.2020.117407

Guo, N., Li, H., Xu, X., \& Yu, H. (2016). Hierarchical Fe 3 O 4 @MoS 2 /Ag 3 PO 4 magnetic nanocomposites: Enhanced and stable photocatalytic performance for water purification under visible light irradiation. Applied Surface Science, 389, 227-239. https://doi.org/10.1016/j.apsusc.2016.07.099

Hao, Y., Li, L., Zhang, J., Luo, H., Zhang, X., \& Chen, E. (2017). Multilayer and open structure of dendritic crosslinked CeO2-ZrO2 composite: Enhanced photocatalytic degradation and water splitting performance. International Journal of Hydrogen Energy, 42(9), 5916-5929. https://doi.org/10.1016/j.ijhydene.2017.01.093

Hernandez, Y., Nicolosi, V., Lotya, M., Blighe, F. M., Sun, Z., De, S., McGovern, I. T., Holland, B., Byrne, M., Gun'ko, Y. K., Boland, J. J., Niraj, P., Duesberg, G., Krishnamurthy, S., Goodhue, R., Hutchison, J., Scardaci, V., Ferrari, A. C., \& Coleman, J. N. (2008). High-yield production of graphene by liquid-phase exfoliation of graphite. Nature Nanotechnology, 3(9), 563-568. https://doi.org/10.1038/nnano.2008.215

Huang, W., Liu, N., Zhang, X., Wu, M., \& Tang, L. (2017). Metal organic framework g-C 3 N 4 /MIL-53(Fe) heterojunctions with enhanced photocatalytic activity for $\mathrm{Cr}(\mathrm{VI})$ reduction under visible light. Applied Surface Science, 425(Vi), 107-116. https://doi.org/10.1016/j.apsusc.2017.07.050

Jawaid, A., Nepal, D., Park, K., Jespersen, M., Qualley, A., Mirau, P., Drummy, L. F., \& Vaia, R. A. (2016). Mechanism for Liquid Phase Exfoliation of MoS2. Chemistry of Materials, 28(1), 337-348. https://doi.org/10.1021/acs.chemmater.5b04224

Jia, F., Sun, K., Yang, B., Zhang, X., Wang, Q., \& Song, S. (2018). Defect-rich molybdenum disulfide as electrode for enhanced capacitive deionization from water. Desalination, 446(August), 21-30. https://doi.org/10.1016/j.desal.2018.08.024

Jia, F., Wang, Q., Wu, J., Li, Y., \& Song, S. (2017). Two-Dimensional Molybdenum Disulfide as a Superb Adsorbent for Removing Hg2+ from Water. ACS Sustainable Chemistry and Engineering, 5(8), 7410-7419. https://doi.org/10.1021/acssuschemeng.7b01880

Jia, T., Kolpin, A., Ma, C., Chau-Ting Chan, R., Kwok, W. M., \& Tsang, S. C. E. (2014). A graphene dispersed CdS-MoS2 nanocrystal ensemble for cooperative photocatalytic hydrogen production from water. Chemical Communications, 50(10), 1185-1188. https://doi.org/10.1039/c3cc47301e

Jiang, J., Wang, H., Chen, X., Li, S., Xie, T., Wang, D., \& Lin, Y. (2017). Enhanced photocatalytic degradation of phenol and photogenerated charges transfer property over BiOI-loaded ZnO composites. Journal of Colloid and Interface Science, 494, 130-138. https://doi.org/10.1016/j.jcis.2017.01.064 
Jo, W. K., Lee, J. Y., \& Selvam, N. C. S. (2016). Synthesis of MoS2 nanosheets loaded ZnO-g-C3N4 nanocomposites for enhanced photocatalytic applications. Chemical Engineering Journal, 289, 306-318. https://doi.org/10.1016/j.cej.2015.12.080

Kolodziejczak-Radzimska, A., \& Jesionowski, T. (2014). Zinc oxide-from synthesis to application: A review. Materials, 7(4), 2833-2881. https://doi.org/10.3390/ma7042833

Kumar, S., Maivizhikannan, V., Drews, J., \& Krishnan, V. (2019). Fabrication of nanoheterostructures of boron doped ZnO-MoS 2 with enhanced photostability and photocatalytic activity for environmental remediation applications. Vacuum, 163(July 2018), 88-98. https://doi.org/10.1016/j.vacuum.2019.02.001

Kumar, S., Sharma, V., Bhattacharyya, K., \& Krishnan, V. (2016). Synergetic effect of MoS2-RGO doping to enhance the photocatalytic performance of ZnO nanoparticles. New Journal of Chemistry, 40(6), 5185-5197. https://doi.org/10.1039/c5nj03595c

Lee, K. M., Lai, C. W., Ngai, K. S., \& Juan, J. C. (2016). Recent developments of zinc oxide based photocatalyst in water treatment technology: A review. In Water Research (Vol. 88). Elsevier Ltd. https://doi.org/10.1016/j.watres.2015.09.045

Lee, S. U., Jun, Y. S., Lee, E. Z., Heo, N. S., Hong, W. H., Huh, Y. S., \& Chang, Y. K. (2015). Selective silver ion adsorption onto mesoporous graphitic carbon nitride. Carbon, 95, 58-64. https://doi.org/10.1016/j.carbon.2015.08.012

Lee, Y. H., Zhang, X. Q., Zhang, W., Chang, M. T., Lin, C. Te, Chang, K. Di, Yu, Y. C., Wang, J. T. W., Chang, C. S., Li, L. J., \& Lin, T. W. (2012). Synthesis of large-area MoS 2 atomic layers with chemical vapor deposition. Advanced Materials, 24(17), 2320-2325. https://doi.org/10.1002/adma.201104798

Li, Haiping, Liu, J., Hu, T., Du, N., Song, S., \& Hou, W. (2016). Synthesis of belt-like BiOBr hierarchical nanostructure with high photocatalytic performance. Materials Research Bulletin, 77, 171-177. https://doi.org/10.1016/j.materresbull.2016.01.039

Li, Honglin, Yu, K., Lei, X., Guo, B., Li, C., Fu, H., \& Zhu, Z. (2015). Synthesis of the MoS2@CuO heterogeneous structure with improved photocatalysis performance and H2O adsorption analysis. Dalton Transactions, 44(22), 10438-10447. https://doi.org/10.1039/c5dt01125f

Li, Honglin, Yu, K., Li, C., Guo, B., Lei, X., Fu, H., \& Zhu, Z. (2015). Novel dual-petal nanostructured WS2@MoS2 with enhanced photocatalytic performance and a comprehensive first-principles investigation. Journal of Materials Chemistry A, 3(40), 20225-20235. https://doi.org/10.1039/c5ta05283a

Li, J., Liu, X., Pan, L., Qin, W., Chen, T., \& Sun, Z. (2014). MoS2-reduced graphene oxide composites synthesized via a microwave-assisted method for visible-light photocatalytic degradation of methylene blue. RSC Advances, 4(19), 9647-9651. https://doi.org/10.1039/c3ra46956e

Li, M., Wang, J., Zhang, P., Deng, Q., Zhang, J., Jiang, K., Hu, Z., \& Chu, J. (2017). Superior adsorption and photoinduced carries transfer behaviors of dandelion-shaped Bi2S3@MoS2: Experiments and theory. Scientific Reports, 7(February), 1-14. https://doi.org/10.1038/srep42484

Li, W., Feng, C., Dai, S., Yue, J., Hua, F., \& Hou, H. (2015). Fabrication of sulfur-doped g-C/Au/CdS Z-scheme photocatalyst to improve the photocatalytic performance under visible light. Applied Catalysis B: Environmental, 168-169(C), 465-471. https://doi.org/10.1016/j.apcatb.2015.01.012

Li, Y., Wu, S., Huang, L., Xu, H., Zhang, R., Qu, M., Gao, Q., \& Li, H. (2015). G-C3N4 modified Bi2O3 composites with enhanced visible-light photocatalytic activity. Journal of Physics and Chemistry of Solids, 76, 112-119. https://doi.org/10.1016/j.jpcs.2014.08.012

Liang, D., Jing, T., Ma, Y., Hao, J., Sun, G., \& Deng, M. (2016). Photocatalytic Properties of g-C6N6/g-C3N4 Heterostructure: A Theoretical Study. Journal of Physical Chemistry C, 120(42), 24023-24029. https://doi.org/10.1021/acs.jpcc.6b08699

Liao, G., Chen, S., Quan, X., Yu, H., \& Zhao, H. (2012). Graphene oxide modified g-C 3N 4 hybrid with enhanced photocatalytic capability under visible light irradiation. Journal of Materials Chemistry, 22(6), 2721-2726. https://doi.org/10.1039/c1jm13490f

Lin, Y. C., Zhang, W., Huang, J. K., Liu, K. K., Lee, Y. H., Liang, C. Te, Chu, C. W., \& Li, L. J. (2012). Wafer-scale MoS2 thin layers prepared by MoO3 sulfurization. Nanoscale, 4(20), 6637-6641. https://doi.org/10.1039/c2nr31833d

Liu, C., Wang, Q., Jia, F., \& Song, S. (2019). Adsorption of heavy metals on molybdenum disulfide in water: A critical review. Journal of Molecular Liquids, 292, 111390. https://doi.org/10.1016/j.molliq.2019.111390

Liu, Chang, Jia, F., Wang, Q., Yang, B., \& Song, S. (2017). Two-dimensional molybdenum disulfide as adsorbent for high-efficient Pb(II) removal from water. Applied Materials Today, 9, 220-228. https://doi.org/10.1016/j.apmt.2017.07.009

Liu, Chunbo, Chen, J., Che, H., Huang, K., Charpentier, P. A., Xu, W. Z., Shi, W., \& Dong, H. J. (2017). Construction and enhanced photocatalytic activities of a hydrogenated TiO2 nanobelt coated with CDs/MoS2 nanosheets. RSC Advances, 7(14), 8429-8442. https://doi.org/10.1039/c6ra28479e

Liu, J., Dong, C., Deng, Y., Ji, J., Bao, S., Chen, C., Shen, B., Zhang, J., \& Xing, M. (2018). Molybdenum sulfide Co-catalytic Fenton reaction for rapid and efficient inactivation of Escherichia coli. Water Research, 145, 312-320. https://doi.org/10.1016/j.watres.2018.08.039

Long, L. L., Chen, J. J., Zhang, X., Zhang, A. Y., Huang, Y. X., Rong, Q., \& Yu, H. Q. (2016). Layer-controlled growth of MoS2 on self-assembled flowerlike Bi2S3 for enhanced photocatalysis under visible light irradiation. NPG Asia Materials, 8(4), e263-9. https://doi.org/10.1038/am.2016.46

Low, J., Cheng, B., \& Yu, J. (2017). Surface modification and enhanced photocatalytic CO 2 reduction performance of TiO 2 : a review. Applied Surface Science, 392, 658-686. https://doi.org/10.1016/j.apsusc.2016.09.093

Lu, X., Jin, Y., Zhang, X., Xu, G., Wang, D., Lv, J., Zheng, Z., \& Wu, Y. (2016). Controllable synthesis of graphitic C3N4/ultrathin MoS2 nanosheet hybrid nanostructures with enhanced photocatalytic performance. Dalton Transactions, 45(39), 15406-15414. https://doi.org/10.1039/c6dt02247b 
Luo, J., Fu, K., Sun, M., Yin, K., Wang, D., Liu, X., \& Crittenden, J. C. (2019). Phase-Mediated Heavy Metal Adsorption from Aqueous Solutions Using Two-Dimensional Layered MoS2. ACS Applied Materials and Interfaces, 11(42), 38789-38797. https://doi.org/10.1021/acsami.9b14019

Ma, C. B., Du, Y., Du, B., Wang, H., \& Wang, E. (2018). Investigation of an eco-friendly aerogel as a substrate for the immobilization of MoS2 nanoflowers for removal of mercury species from aqueous solutions. Journal of Colloid and Interface Science, 525, 251-259. https://doi.org/10.1016/j.jcis.2018.04.079

Ma, L., Xu, L. M., Xu, X. Y., Luo, Y. L., \& Chen, W. X. (2009). Synthesis and characterization of flower-like MoS2 microspheres by a facile hydrothermal route. Materials Letters, 63(23), 2022-2024. https://doi.org/10.1016/j.matlet.2009.06.039

Mak, K. F., Lee, C., Hone, J., Shan, J., \& Heinz, T. F. (2010). Atomically thin MoS2: A new direct-gap semiconductor. Physical Review Letters, 105(13), 2-5. https://doi.org/10.1103/PhysRevLett.105.136805

Mário, E. D. A., Liu, C., Ezugwu, C. I., Mao, S., Jia, F., \& Song, S. (2020). Molybdenum disulfide/montmorillonite composite as a highly efficient adsorbent for mercury removal from wastewater. Applied Clay Science, 184(November 2019), 105370. https://doi.org/10.1016/j.clay.2019.105370

Midya, A., Ghorai, A., Mukherjee, S., Maiti, R., \& Ray, S. K. (2016). Hydrothermal growth of few layer 2H-MoS2 for heterojunction photodetector and visible light induced photocatalytic applications. Journal of Materials Chemistry A, 4(12), 4534-4543. https://doi.org/10.1039/c5ta09003b

Mousavi, M., \& Habibi-Yangjeh, A. (2016). Magnetically separable ternary g-C3N4/Fe3O4/BiOI nanocomposites: Novel visible-light-driven photocatalysts based on graphitic carbon nitride. Journal of Colloid and Interface Science, 465, 83-92. https://doi.org/10.1016/j.jcis.2015.11.057

Najmaei, S., Liu, Z., Zhou, W., Zou, X., Shi, G., Lei, S., Yakobson, B. I., Idrobo, J. C., Ajayan, P. M., \& Lou, J. (2013). Vapour phase growth and grain boundary structure of molybdenum disulphide atomic layers. Nature Materials, 12(8), 754-759. https://doi.org/10.1038/nmat3673

Ni, Z., Sun, Y., Zhang, Y., \& Dong, F. (2016). Fabrication, modification and application of (BiO) 2 CO 3 -based photocatalysts: A review. Applied Surface Science, 365(2016), 314-335. https://doi.org/10.1016/j.apsusc.2015.12.231

Nitayaphat, W., \& Jintakosol, T. (2015). Removal of silver(I) from aqueous solutions by chitosan/bamboo charcoal composite beads. Journal of Cleaner Production, 87(1), 850-855. https://doi.org/10.1016/j.jclepro.2014.10.003

Pan, L., Liu, X., Sun, Z., \& Sun, C. Q. (2013). Nanophotocatalysts via microwave-assisted solution-phase synthesis for efficient photocatalysis. Journal of Materials Chemistry A, 1(29), 8299-8326. https://doi.org/10.1039/c3ta10981j

Pearson, R. G. (1968). Hard and soft acids and bases, HSAB, part 1: Fundamental principles. Journal of Chemical Education, 45(9), 581. https://doi.org/10.1021/ed045p581

Peng, W. C., \& Li, X. Y. (2014). Synthesis of MoS2/g-C3N4 as a solar light-responsive photocatalyst for organic degradation. Catalysis Communications, 49, 63-67. https://doi.org/10.1016/j.catcom.2014.02.008

Peng, W., Li, H., Liu, Y., \& Song, S. (2017). A review on heavy metal ions adsorption from water by graphene oxide and its composites. Journal of Molecular Liquids, 230, 496-504. https://doi.org/10.1016/j.molliq.2017.01.064

Peng, Y., Meng, Z., Zhong, C., Lu, J., Yang, Z., \& Qian, Y. (2002). Tube- and ball-like amorphous MoS2 prepared by a solvothermal method. Materials Chemistry and Physics, 73(2-3), 327-329. https://doi.org/10.1016/S0254-0584(01)00364-9

Qi, Y., Luan, Y., Yang, M., Wang, G., Tan, L., \& Li, J. (2014). Alkali concentration-dependent tailoring of highly controllable titanate nanostructures: From yolk-shell, hollow 3D nanospheres to 1D nanowires. Applied Surface Science, 293, 359-365. https://doi.org/10.1016/j.apsusc.2013.12.170

Qian, W., Greaney, P. A., Fowler, S., Chiu, S. K., Goforth, A. M., \& Jiao, J. (2014). Low-temperature nitrogen doping in ammonia solution for production of $\mathrm{N}$-doped TiO2-hybridized graphene as a highly efficient photocatalyst for water treatment. ACS Sustainable Chemistry and Engineering, 2(7), 1802-1810. https://doi.org/10.1021/sc5001176

Qiu, J., Zheng, W., Yuan, R., Yue, C., Li, D., Liu, F., \& Zhu, J. (2020). A novel 3D nanofibrous aerogel-based MoS2@ Co3S4 heterojunction photocatalyst for water remediation and hydrogen evolution under simulated solar irradiation. Applied Catalysis B: Environmental, 264(November 2019), 118514. https://doi.org/10.1016/j.apcatb.2019.118514

Renuka, L., Anantharaju, K. S., Vidya, Y. S., Nagaswarupa, H. P., Prashantha, S. C., Sharma, S. C., Nagabhushana, H., \& Darshan, G. P. (2017). A simple combustion method for the synthesis of multi-functional $\mathrm{ZrO} 2 / \mathrm{CuO}$ nanocomposites: Excellent performance as Sunlight photocatalysts and enhanced latent fingerprint detection. Applied Catalysis B: Environmental, 210, 97-115. https://doi.org/10.1016/j.apcatb.2017.03.055

Shahzad, A., Jang, J., Lim, S. R., \& Lee, D. S. (2020). Unique selectivity and rapid uptake of molybdenum-disulfide-functionalized MXene nanocomposite for mercury adsorption. Environmental Research, 182(September 2019), 109005. https://doi.org/10.1016/j.envres.2019.109005

Shi, Y., Zhou, W., Lu, A. Y., Fang, W., Lee, Y. H., Hsu, A. L., Kim, S. M., Kim, K. K., Yang, H. Y., Li, L. J., Idrobo, J. C., \& Kong, J. (2012). Van der Waals epitaxy of MoS 2 layers using graphene as growth templates. Nano Letters, 12(6), 2784-2791. https://doi.org/10.1021/nl204562j

Smith, R. J., King, P. J., Lotya, M., Wirtz, C., Khan, U., De, S., O’Neill, A., Duesberg, G. S., Grunlan, J. C., Moriarty, G., Chen, J., Wang, J., Minett, A. I., Nicolosi, V., \& Coleman, J. N. (2011). Large-scale exfoliation of inorganic layered compounds in aqueous surfactant solutions. Advanced Materials, 23(34), 3944-3948. https://doi.org/10.1002/adma.201102584

Song, H. J., You, S., Jia, X. H., \& Yang, J. (2015). MoS2 nanosheets decorated with magnetic Fe3O4 nanoparticles and their ultrafast adsorption for wastewater treatment. Ceramics International, 41(10), 13896-13902. https://doi.org/10.1016/j.ceramint.2015.08.023

Su, J., Bi, L., Wang, C., Lyu, T., \& Pan, G. (2019). Enhancement of cadmium removal by oxygen-doped carbon nitride with molybdenum and sulphur hybridization. Journal of Colloid and Interface Science, 556, 606-615. https://doi.org/10.1016/j.jcis.2019.08.104 
Sun, T., Zhao, Z., Liang, Z., Liu, J., Shi, W., \& Cui, F. (2017). Efficient removal of arsenite through photocatalytic oxidation and adsorption by ZrO 2 -Fe 3 O 4 magnetic nanoparticles. Applied Surface Science, 416, 656-665. https://doi.org/10.1016/j.apsusc.2017.04.137

Tan, X., Kang, W., Liu, J., \& Zhang, C. (2019). Synergistic Exfoliation of MoS2 by Ultrasound Sonication in a Supercritical Fluid Based Complex Solvent. Nanoscale Research Letters, 14(1). https://doi.org/10.1186/s11671-019-3126-4

Tan, Y. H., Yu, K., Li, J. Z., Fu, H., \& Zhu, Z. Q. (2014). MoS2@ZnO nano-heterojunctions with enhanced photocatalysis and field emission properties. Journal of Applied Physics, 116(6). https://doi.org/10.1063/1.4893020

Tang, L., Jia, C. tao, Xue, Y. cheng, Li, L., Wang, A. qi, Xu, G., Liu, N., \& Wu, M. hong. (2017). Fabrication of compressible and recyclable macroscopic gC3N4/GO aerogel hybrids for visible-light harvesting: A promising strategy for water remediation. Applied Catalysis B: Environmental, 219, 241-248, https://doi.org/10.1016/j.apcatb.2017.07.053

Tisseraud, C., Comminges, C., Pronier, S., Pouilloux, Y., \& Le Valant, A. (2016). The $\mathrm{Cu}-\mathrm{ZnO}$ synergy in methanol synthesis Part 3: Impact of the composition of a selective $\mathrm{Cu} @ \mathrm{ZnOx}$ core-shell catalyst on methanol rate explained by experimental studies and a concentric spheres model. Journal of Catalysis, 343, 106-114. https://doi.org/10.1016/j.jcat.2015.12.005

Tong, S., Deng, H., Wang, L., Huang, T., Liu, S., \& Wang, J. (2018). Multi-functional nanohybrid of ultrathin molybdenum disulfide nanosheets decorated with cerium oxide nanoparticles for preferential uptake of lead (II) ions. Chemical Engineering Journal, 335(August 2017), 22-31. https://doi.org/10.1016/j.cej.2017.10.056

Vassileva, P., Tzvetkova, P., Lakov, L., \& Peshev, O. (2008). Thiouracil modified activated carbon as a sorbent for some precious and heavy metal ions. Journal of Porous Materials, 15(5), 593-599. https://doi.org/10.1007/s10934-007-9138-y

Vattikuti, S. V. P., \& Byon, C. (2016). Bi2S3 nanorods embedded with MoS2 nanosheets composite for photodegradation of phenol red under visible light irradiation. Superlattices and Microstructures, 100, 514-525. https://doi.org/10.1016/j.spmi.2016.10.012

Wan, J., Du, X., Liu, E., Hu, Y., Fan, J., \& Hu, X. (2017). Z-scheme visible-light-driven Ag3PO4nanoparticle@MoS2quantum dot/few-layered MoS2nanosheet heterostructures with high efficiency and stability for photocatalytic selective oxidation. Journal of Catalysis, 345, $281-294$. https://doi.org/10.1016/j.jcat.2016.11.013

Wang, H., Bai, Y., Yang, J., Lang, X., Li, J., \& Guo, L. (2012). A facile way to rejuvenate Ag 3PO 4 as a recyclable highly efficient photocatalyst. Chemistry A European Journal, 18(18), 5524-5529. https://doi.org/10.1002/chem.201103189

Wang, Q., Yun, G., Bai, Y., An, N., Lian, J., Huang, H., \& Su, B. (2014). Photodegradation of rhodamine B with MoS 2 /Bi 2 O 2 CO 3 composites under UV light irradiation. Applied Surface Science, 313, 537-544. https://doi.org/10.1016/j.apsusc.2014.06.018

Wang, Zhen, Chen, T., Chen, W., Chang, K., Ma, L., Huang, G., Chen, D., \& Lee, J. Y. (2013). CTAB-assisted synthesis of single-layer MoS2-graphene composites as anode materials of Li-ion batteries. Journal of Materials Chemistry A, 1(6), 2202-2210. https://doi.org/10.1039/c2ta00598k

Wang, Zhongying, \& Mi, B. (2017). Environmental Applications of 2D Molybdenum Disulfide (MoS2) Nanosheets. Environmental Science and Technology, 51(15), 8229-8244. https://doi.org/10.1021/acs.est.7b01466

Wang, Zhongying, Sim, A., Urban, J. J., \& Mi, B. (2018). Removal and Recovery of Heavy Metal Ions by Two-dimensional MoS2 Nanosheets: Performance and Mechanisms. Environmental Science and Technology, 52(17), 9741-9748. https://doi.org/10.1021/acs.est.8b01705

Wang, Zhongying, Zhu, W., Qiu, Y., Yi, X., Von Dem Bussche, A., Kane, A., Gao, H., Koski, K., \& Hurt, R. (2016). Biological and environmental interactions of emerging two-dimensional nanomaterials. Chemical Society Reviews, 45(6), 1750-1780. https://doi.org/10.1039/c5cs00914f

Wang, Zongwu, Zhang, J., Wen, T., Liu, X., Wang, Y., Yang, H., Sun, J., Feng, J., Dong, S., \& Sun, J. (2020). Highly effective remediation of Pb(II) and $\mathrm{Hg}$ (II) contaminated wastewater and soil by flower-like magnetic MoS2 nanohybrid. Science of the Total Environment, 699, 134341. https://doi.org/10.1016/j.scitotenv.2019.134341

Wu, M. hong, Li, L., Liu, N., Wang, D. jin, Xue, Y. cheng, \& Tang, L. (2018). Molybdenum disulfide (MoS2) as a co-catalyst for photocatalytic degradation of organic contaminants: A review. Process Safety and Environmental Protection, 118, 40-58. https://doi.org/10.1016/j.psep.2018.06.025

Wu, P., Wu, W., Li, S., Xing, N., Zhu, N., Li, P., Wu, J., Yang, C., \& Dang, Z. (2009). Removal of Cd2+ from aqueous solution by adsorption using Femontmorillonite. Journal of Hazardous Materials, 169(1-3), 824-830. https://doi.org/10.1016/j.jhazmat.2009.04.022

Xiang, Q., Cheng, B., \& Yu, J. (2015). Graphene-Based Photocatalysts for Solar-Fuel Generation. Angewandte Chemie - International Edition, 54(39), 1135011366. https://doi.org/10.1002/anie.201411096

Xiang, Z., Wang, Y., Zhang, D., \& Ju, P. (2016). BiOI/BiVO4 p-n heterojunction with enhanced photocatalytic activity under visible-light irradiation. Journal of Industrial and Engineering Chemistry, 40, 83-92. https://doi.org/10.1016/j.jiec.2016.06.009

Xie, J., Zhang, J., Li, S., Grote, F., Zhang, X., Zhang, H., Wang, R., Lei, Y., Pan, B., \& Xie, Y. (2014). Erratum: Controllable disorder engineering in oxygenincorporated MoS 2 ultrathin nanosheets for efficient hydrogen evolution (Journal of the American Chemical Society (2013) 135 (17881-17888)). Journal of the American Chemical Society, 136(4), 1680. https://doi.org/10.1021/ja4129636

Xiong, F., Zhang, J., Zhu, Z., Yuan, X., \& Qin, S. (2015). Ultrabroadband, More than One Order Absorption Enhancement in Graphene with Plasmonic Light Trapping. Scientific Reports, 5(November), 1-8. https://doi.org/10.1038/srep16998

Xiong, X., Ding, L., Wang, Q., Li, Y., Jiang, Q., \& Hu, J. (2016). Synthesis and photocatalytic activity of BiOBr nanosheets with tunable exposed (0 10$)$ facets. Applied Catalysis B: Environmental, 188, 283-291. https://doi.org/10.1016/j.apcatb.2016.02.018 
Research, Society and Development, v. 10, n. 3, e12410313187, 2021

(CC BY 4.0) | ISSN 2525-3409 | DOI: http://dx.doi.org/10.33448/rsd-v10i3.13187

Xu, C., Xu, B., Gu, Y., Xiong, Z., Sun, J., \& Zhao, X. S. (2013). Graphene-based electrodes for electrochemical energy storage. Energy and Environmental Science, 6(6), 1388-1414. https://doi.org/10.1039/c3ee23870a

Yi-Zhu, P., Wan-Hong, M., Man-Ke, J., Xiao-Rong, Z., Johnson, D. M., \& Ying-Ping, H. (2016). Comparing the degradation of acetochlor to RhB using $\mathrm{BiOBr}$ under visible light: A significantly different rate-catalyst dose relationship. Applied Catalysis B: Environmental, 181, 517-523. https://doi.org/10.1016/j.apcatb.2015.08.002

Yuan, Y. J., Ye, Z. J., Lu, H. W., Hu, B., Li, Y. H., Chen, D. Q., Zhong, J. S., Yu, Z. T., \& Zou, Z. G. (2016). Constructing Anatase TiO2 Nanosheets with Exposed (001) Facets/Layered MoS2 Two-Dimensional Nanojunctions for Enhanced Solar Hydrogen Generation. ACS Catalysis, 6(2), 532-541. https://doi.org/10.1021/acscatal.5b02036

Yuan, Y., Shen, P., Li, Q., Chen, G., Zhang, H., Zhu, L., Zou, B., \& Liu, B. (2017). Excellent photocatalytic performance of few-layer MoS2/graphene hybrids. Journal of Alloys and Compounds, 700, 12-17. https://doi.org/10.1016/j.jallcom.2017.01.027

Yusan, S., Gok, C., Erenturk, S., \& Aytas, S. (2012). Adsorptive removal of thorium (IV) using calcined and flux calcined diatomite from Turkey: Evaluation of equilibrium, kinetic and thermodynamic data. Applied Clay Science, 67-68, 106-116. https://doi.org/10.1016/j.clay.2012.05.012

Zeng, Z., Yin, Z., Huang, X., Li, H., He, Q., Lu, G., Boey, F., \& Zhang, H. (2011). Single-Layer Semiconducting Nanosheets: High-Yield Preparation and Device Fabrication. Angewandte Chemie, 123(47), 11289-11293. https://doi.org/10.1002/ange.201106004

Zhang, G., Huang, C., \& Wang, X. (2015). Dispersing molecular cobalt in graphitic carbon nitride frameworks for photocatalytic water oxidation. Small, 11(9-10), 1215-1221. https://doi.org/10.1002/smll.201402636

Zhang, J. J., Gao, B., \& Dong, S. (2016). Strain-enhanced superconductivity of MoX2(X=S-or Se) bilayers with Na intercalation. Physical Review B, 93(15), 1-6. https://doi.org/10.1103/PhysRevB.93.155430

Zheng, Y., Zhang, W., Li, Y., Chen, J., Yu, B., Wang, J., Zhang, L., \& Zhang, J. (2017). Energy related CO2 conversion and utilization: Advanced materials/nanomaterials, reaction mechanisms and technologies. Nano Energy, 40, 512-539. https://doi.org/10.1016/j.nanoen.2017.08.049

Zhong, X. L., \& Li, Z. Y. (2012). Giant enhancement of near-ultraviolet light absorption by TiO 2 via a three-dimensional aluminum plasmonic nano funnelantenna. Journal of Physical Chemistry C, 116(40), 21547-21555. https://doi.org/10.1021/jp306562u

Zhou, G., Xu, X., Yu, J., Feng, B., Zhang, Y., Hu, J., \& Zhou, Y. (2014). Vertically aligned MoS2/MoOxheterojunction nanosheets for enhanced visible-light photocatalytic activity and photostability. CrystEngComm, 16(38), 9025-9032. https://doi.org/10.1039/c4ce01169d

Zhu, C., Zhang, L., Jiang, B., Zheng, J., Hu, P., Li, S., Wu, M., \& Wu, W. (2016). Fabrication of Z-scheme Ag 3 PO 4 /MoS 2 composites with enhanced photocatalytic activity and stability for organic pollutant degradation. Applied Surface Science, 377, 99-108. https://doi.org/10.1016/j.apsusc.2016.03.143

Zou, X., Dong, Y., Zhang, X., Cui, Y., Ou, X., \& Qi, X. (2017). The highly enhanced visible light photocatalytic degradation of gaseous o-dichlorobenzene through fabricating like-flowers $\mathrm{BiPO} 4 / \mathrm{BiOBr} \mathrm{p}-\mathrm{n}$ heterojunction composites. Applied Surface Science, 391, 525-534. https://doi.org/10.1016/j.apsusc.2016.06.003 